\title{
REGULARITY THEORY AND SUPERALGEBRAIC SOLVERS FOR WIRE ANTENNA PROBLEMS*
}

\author{
OSCAR P. BRUNO ${ }^{\dagger}$ AND MICHAEL C. HASLAM ${ }^{\dagger}$
}

\begin{abstract}
We consider the problem of evaluating the current distribution $J(z)$ that is induced on a straight wire antenna by a time-harmonic incident electromagnetic field. The scope of this paper is twofold. One of its main contributions is a regularity proof for a straight wire occupying the interval $[-1,1]$. In particular, for a smooth time-harmonic incident field this theorem implies that $J(z)=I(z) / \sqrt{1-z^{2}}$, where $I(z)$ is an infinitely differentiable function- the previous state of the art in this regard placed $I$ in the Sobolev space $W^{1, p}, p>1$. The second focus of this work is on numerics: we present three superalgebraically convergent algorithms for the solution of wire problems, two based on Hallén's integral equation and one based on the Pocklington integrodifferential equation. Both our proof and our algorithms are based on two main elements: (1) a new decomposition of the kernel of the form $G(z)=F_{1}(z) \ln |z|+F_{2}(z)$, where $F_{1}(z)$ and $F_{2}(z)$ are analytic functions on the real line; and (2) removal of the end-point square root singularities by means of a coordinate transformation. The Hallén- and Pocklington-based algorithms we propose converge superalgebraically: faster than $\mathcal{O}\left(N^{-m}\right)$ and $\mathcal{O}\left(M^{-m}\right)$ for any positive integer $m$, where $N$ and $M$ are the numbers of unknowns and the number of integration points required for construction of the discretized operator, respectively. In previous studies, at most the leading-order contribution to the logarithmic singular term was extracted from the kernel and treated analytically, the higher-order singular derivatives were left untreated, and the resulting integration methods for the kernel exhibit $\mathcal{O}\left(M^{-3}\right)$ convergence at best. A rather comprehensive set of tests we consider shows that, in many cases, to achieve a given accuracy, the numbers $N$ of unknowns required by our codes are up to a factor of five times smaller than those required by the best solvers previously available; the required number $M$ of integration points, in turn, can be several orders of magnitude smaller than those required in previous methods. In particular, four-digit solutions were found in computational times of the order of four seconds and, in most cases, of the order of a fraction of a second on a contemporary personal computer; much higher accuracies result in very small additional computing times.
\end{abstract}

Key words. electromagnetic scattering, wire antenna, Pocklington, Hallén

AMS subject classifications. $65-02,78-02$

DOI. $10.1137 / 050648262$

1. Introduction. The problem of evaluating the current distribution that is induced on a thin straight wire antenna by a given time-harmonic incident electromagnetic field was first described by Pocklington [10] more than a century ago; its computation remains an important and difficult engineering problem to this day. Once such currents have been calculated, the fields scattered by the wire can easily be produced by integration. The current $J(z)$ induced on a thin wire of radius $a$ by an incident electromagnetic field with wave number $k$ and axial component $e(z)$ can be obtained as a solution of the Pocklington equation

$$
\left(\frac{\partial^{2}}{\partial z^{2}}+k^{2}\right) \int_{-1}^{1} G(z-t) J(t) d t=-4 \pi i k e(z)
$$

* Received by the editors December 22, 2005; accepted for publication (in revised form) December 27, 2006; published electronically June 12, 2007. This work was supported in part by the Air Force Office of Scientific Research, the National Science Foundation, and the National Aeronautics and Space Administration.

http://www.siam.org/journals/sisc/29-4/64826.html

$\dagger$ Applied and Computational Mathematics, California Institute of Technology, Pasadena, CA 91125 (bruno@acm.caltech.edu, mchaslam@acm.caltech.edu). The second author was supported by the Natural Sciences and Engineering Research Council of Canada. 
subject to the end-point conditions

$$
J(-1)=J(1)=0 .
$$

An alternative (and, in exact arithmetic, equivalent) formulation for this problem is given by the Hallén equation, which results from (1.1) by inverting the Helmholtz operator. The Hallén equation thus reads

$$
\int_{-1}^{1} G(z-t) J(t) d t=\alpha_{1} \cos k z+\alpha_{2} \sin k z-4 \pi i \int_{-1}^{z} e(t) \sin [k(z-t)] d t,
$$

where $\alpha_{1}$ and $\alpha_{2}$ are constants which are chosen so that the current distribution satisfies the end-point conditions (1.2). The kernel $G(z)$ in these equations, which is given by

$$
G(z)=\frac{1}{\pi} \int_{0}^{\pi} \frac{\mathrm{e}^{i k \sqrt{z^{2}+4 a^{2} \sin ^{2} \psi}}}{\sqrt{z^{2}+4 a^{2} \sin ^{2} \psi}} d \psi,
$$

has a logarithmic singularity at $z=0$; see section 2 .

The scope of this paper is twofold. One of its main contributions is a regularity proof: expressing the current in the form $J(z)=I(z) / \sqrt{1-z^{2}}$ (which has been known since the work by Jones [7] and Rynne [13]), our result relates the regularity of the function $I(z)$ to that of the incident field. In particular, for an infinitely differentiable incident field our theorem implies that the "reduced current" $I(z)$ is infinitely differentiable as well - the previous state of the art in this regard placed $I$ in the Sobolev space $W^{1, p}, p>1$. The second focus of this work is on numerics: we present three superalgebraically convergent algorithms for the solution of wire scattering problems, two based on Hallén's integral equation and one based on the Pocklington integrodifferential equation. To achieve superalgebraic convergence, these algorithms rely on a number of techniques which address specifically the difficulties posed by wire scattering problems.

Some of the concepts we present in this paper are used in both our theoretical and our numerical treatments; others are specifically designed to tackle numerical issues presented by the problem at hand. In particular, to both establish our regularity result and produce algorithms of high-order accuracy for this problem, we do the following:

1. Introduce a new decomposition of the kernel, of the form

$$
G(z)=F_{1}(z) \ln |z|+F_{2}(z),
$$

where $F_{1}(z)$ and $F_{2}(z)$ are analytic functions on the real line. Albeit analytic, the functions $F_{i}$ exhibit a rather interesting "nearly singular" behavior.

2. Utilize a coordinate transformation of the form $z=\cos (\theta)$. This transformation, which is essential for our theoretical discussion (section 3), is also quite useful from a numerical standpoint, since it simultaneously regularizes the integration problem and leads to a Chebyshev representation of the reduced current $I$.

To obtain accurate and efficient algorithms we introduce additional numerical strategies. In particular:

3. For very thin wires we use the transformation $z=2 \cos w /\left(1+\cos ^{2} w\right)$ instead of the one mentioned in point 2 above. The $w \rightarrow z$ transformation results 
in significantly improved performance for very thin wires (see points (a), (b), and (c) in section 5.1): instead of the quadratic $z$-clustering introduced at $z= \pm 1$ by the $\theta \rightarrow z$ transformation, the $w \rightarrow z$ change of variables introduces quartic point clustering around the end-points. This additional refinement resolves a near singularity that occurs in the reduced current $I$ for $a$ very close to zero.

4. To produce well-conditioned linear systems we discretize our integral equations by sampling them at $z$ points corresponding to equispaced $\theta$ or $w$ grids, depending on whether the $\theta \rightarrow z$ or the $w \rightarrow z$ transformation is used. A sampling of the integral operators at regular $z$ grids gives rise to highly illconditioned linear algebra problems.

5. The integration algorithm itself, on the other hand, utilizes (i) a localized integration scheme based on use of fine and coarse Chebyshev grids for integration of highly peaked integrands that arise from corresponding (smooth) peaks in the functions $F_{1}$ and $F_{2}$, as well as (ii) a new weighted-quadrature formula for log-Chebyshev integrals (see Appendix B).

6. For efficiency, the kernel evaluations are based on a new asymptotic formula (equation (2.20)) which we use for sufficiently large values of a certain dimensionless version $\rho$ of the spatial variable, together with numerical procedures based on expansions and integral representations for the rapid and accurate evaluation of the newly introduced functions $F_{1}$ and $F_{2}$.

Having accounted to all orders for all integrand singularities and near-singularities and in view of our regularity result, we find that the Hallén- and Pocklington-based algorithms we propose converge superalgebraically: faster than $\mathcal{O}\left(N^{-m}\right)$ and $\mathcal{O}\left(M^{-m}\right)$ for any positive integer $m$, where $N$ and $M$ are the numbers of unknowns and the number of integration points required for construction of the discretized operator (i.e., the linear system's matrix), respectively. Previous solution techniques for this problem, in contrast, have not taken into account the precise nature of the kernel's singularity - which, to our knowledge, had not been established before the present work. In many contributions, furthermore, the end-point singularities of the current distribution were not fully accounted for. We show, albeit not straightforward, that it is very advantageous to account for all singularities to high order: as we show in a variety of examples in section 5 , in many cases, to achieve a given accuracy, the numbers $N$ of unknowns required by our codes are as much as five times smaller than those required by the best solvers previously available. The required number $M$ of integration points, in turn, can be several orders of magnitude smaller than those required by previous methods; see paragraph (d) below.

A few comments are in order with regards to the kernel mentioned above and its relationship to various expressions available in the literature:

(a) The normalization of the kernel (1.4) coincides with that of Jones [7] and Rynne [13]; the normalization employed by Davies, Duncan, and Funken [4], on the other hand, includes an additional multiple of $1 /(4 \pi)$, which, here, is incorporated on the right-hand side of (1.1) instead.

(b) Another kernel for the equations above, which results from use of approximations additional to those implicit in (1.1), (1.3), and (1.4), is the so-called reduced kernel

$$
G_{r e d}(z)=\frac{\mathrm{e}^{i k \sqrt{z^{2}+a^{2}}}}{\sqrt{z^{2}+a^{2}}} .
$$

We will not utilize this kernel: as pointed out in [4] and [5], it is not possible 
to obtain reliable results using the reduced kernel $G_{r e d}(z)$ in place of $G(z)$ in (1.1) or (1.3).

(c) In a concise paper [16], Wang derives the expansion

$$
G(z)=\frac{\mathrm{e}^{i k \sqrt{z^{2}+2 a^{2}}}}{\sqrt{z^{2}+2 a^{2}}}+i k \sum_{m=1}^{\infty} \frac{(k a)^{4 m}(2 m-1) ! !}{(2 m) !(2 m) ! !} \frac{h_{2 m}^{(1)}\left(k \sqrt{z^{2}+2 a^{2}}\right)}{k^{2 m}\left(z^{2}+2 a^{2}\right)^{m}}
$$

for the exact straight wire kernel, where $h_{m}^{(1)}(z)$ is a spherical Hankel function of the first kind [1]. This expansion is particularly useful for values of $z$ far away from the origin, for which the series in (1.7) converges rapidly. Unfortunately, this expansion does not seem as useful for values of $z$ close to $z=0$, since the asymptotic behavior of the kernel in this important region cannot be easily produced from (1.7).

(d) It has been known for some time that the kernel (1.4) has the form

$$
G(z)=-\frac{1}{a \pi} \ln |z|+G_{1}(z)
$$

where the function $G_{1}(z)$ is continuous, it has a bounded derivative and an unbounded second derivative; see [7, p. 115], [13], or section 2.2 below. The logarithmic behavior of the function $G(z)$ can be easily grasped by consideration of the expression

$$
G(z)=\frac{2}{\pi \sqrt{z^{2}+4 a^{2}}} K\left(\frac{2 a}{\sqrt{z^{2}+4 a^{2}}}\right)-\frac{1}{\pi} \int_{0}^{\pi} \frac{1-\mathrm{e}^{i k \sqrt{z^{2}+4 a^{2} \sin ^{2} \psi}}}{\sqrt{z^{2}+4 a^{2} \sin ^{2} \psi}} d \psi
$$

where $K$, the complete elliptic integral of the first kind [3], contains the leading-order singularity. Equations (1.8) and (1.9) have been used as the basis for many numerical solvers for Pocklington and Hallén problems $[4,9]$. Numerical integration schemes for integrands containing such singularities which, based on polynomial interpolation, do not explicitly account for the singularity of the second derivative of the integrand typically exhibit loworder convergence. Indeed, the leading singular term in $G_{1}(z)$ is of the form $z^{2} \ln |z|[9]$. The error that results as an $n$th degree Newton-Cotes scheme (e.g., Simpson's rule for $n=2$ ) is used to integrate such a function is of the order of $\mathcal{O}\left(h^{3}\right)$ for all $n \geq 2$, where $h$ is the integration mesh size. The decomposition (1.5) we introduce extends the result embodied in (1.8) and allows for superalgebraic evaluation of the kernel and its integrals; see section 2.3 and Appendix B, respectively. In particular, efficient algorithms for the evaluation of the functions $F_{1}$ and $F_{2}$ can be found in section 2.3.

This paper is organized as follows: After introducing the new decomposition for the exact kernel in section 2, we discuss in section 3 the regularity properties of the current $J(z)$. Our approach to the discretization of the Pocklington and Hallén integral equations is then described in section 4 and Appendix B. A variety of numerical results and conclusions, finally, are presented in section 5 .

2. New decomposition for the exact kernel. In this section we present a derivation of the decomposition (1.5) together with explicit expressions and algorithms for fast and accurate evaluation of the analytic functions $F_{1}$ and $F_{2}$. 
2.1. Taylor expansion of the integrand's exponential. Substituting the exponential in (1.4) by its Taylor expansion and interchanging the order of summation and integration, we obtain

$$
G(z)=g(\rho) / a, \quad \rho=z /(2 a),
$$

where, defining

$$
\begin{gathered}
B_{m}(\rho)=\int_{0}^{\pi}\left(\rho^{2}+\sin ^{2} \psi\right)^{m / 2} d \psi, \\
g_{r}(\rho)=\frac{1}{2 \pi} \sum_{n=0}^{\infty} \frac{(-1)^{n}(2 k a)^{2 n}}{(2 n) !} B_{2 n-1}(\rho) \quad \text { and } \\
g_{i}(\rho)=\frac{1}{2 \pi} \sum_{n=0}^{\infty} \frac{(-1)^{n}(2 k a)^{2 n+1}}{(2 n+1) !} B_{2 n}(\rho),
\end{gathered}
$$

the dimensionless function $g$ is given by

$$
g(\rho)=g_{r}(\rho)+i g_{i}(\rho)
$$

For $m=2 n$ ( $n$ a nonnegative integer), the integrals in (2.2) define a set of polynomials. In particular, calling

$$
R=\sqrt{\rho^{2}+1},
$$

we have the expression

$$
B_{2 n}(\rho)=\pi \sum_{m=0}^{n} \frac{(-1)^{m}}{2^{2 m}}\left(\begin{array}{c}
n \\
m
\end{array}\right)\left(\begin{array}{c}
2 m \\
m
\end{array}\right) R^{2 n-2 m},
$$

which follows from the hypergeometric function representation of the integral in (2.2) [14, eqn. IV(2)] and the series expansion for these functions [1, eqn. 15.4.1]. Using (2.6) and reversing the order of summation in the second equation of (2.3) we obtain an exponentially convergent series for the imaginary part of the kernel: denoting by $j_{n}(z)$ the spherical Bessel function of the first kind [1], our expansion reads

$$
g_{i}(\rho)=k a \sum_{n=0}^{\infty} \frac{1}{2^{2 n} n !}\left(\begin{array}{c}
2 n \\
n
\end{array}\right)\left(\frac{k a}{R}\right)^{n} j_{n}(2 k a R) .
$$

A corresponding closed-form result can be obtained for the real part of the kernel as well: for the values $m=2 n-1$ ( $n$ a nonnegative integer) relevant in this case, the integrals in (2.2) are related to the associated Legendre functions of the second kind, $Q_{\nu}^{\mu}(z)$, of degree $\nu$ and order $\mu$. It follows directly from the integral representation for the functions $Q_{m-1 / 2}^{\mu}(z)[14$, eqn. $\operatorname{VI}(73 c)]$ that

$$
B_{2 n-1}(\rho)=\frac{2}{\sqrt{\pi}} \Gamma(1 / 2-n)(|\rho| R)^{n} Q_{-1 / 2}^{n}\left(2 \rho^{2}+1\right) .
$$


2.2. Decomposition of the real part of the kernel. To make explicit the singular nature of the kernel $g_{r}(\rho)$, we use (2.3) and (2.8) together with known formulae for the Legendre functions of integer order $Q_{\nu}^{n}(z)$. In particular, from [14, eqn. $\left.\mathrm{VI}\left(46^{\prime}\right)\right]$ and using the notation (2.5), we obtain

$Q_{-1 / 2}^{n}\left(2 \rho^{2}+1\right)=-P_{-1 / 2}^{n}\left(2 \rho^{2}+1\right) \ln \left(\frac{|\rho|}{R}\right)+\frac{\sqrt{\pi}}{2 R}\left(\frac{|\rho|}{R}\right)^{n} \Gamma(n+1 / 2) F_{-1 / 2}^{n}\left(2 \rho^{2}+1\right)$,

where $P_{\nu}^{\mu}(z)$ is the Legendre function of the first kind of degree $\nu$ and order $\mu$. Using a slightly different notation from that of [14] we have set

$$
\begin{aligned}
F_{-1 / 2}^{n}\left(2 \rho^{2}+1\right)=\frac{2}{\pi}(-1)^{n} & {\left[\sum_{m=1}^{n} \frac{1}{2 m} \frac{\phi_{n-m}}{\phi_{m}}\left(\frac{R}{\rho}\right)^{2 m}\right.} \\
& \left.+\frac{1}{2} \sum_{m=0}^{\infty}\left(\beta_{m}+\beta_{m+n}\right) \phi_{m} \phi_{m+n}\left(\frac{\rho}{R}\right)^{2 m}\right],
\end{aligned}
$$

where

$$
\phi_{\ell}=\frac{1}{2^{2 \ell}}\left(\begin{array}{c}
2 \ell \\
\ell
\end{array}\right)=\frac{1 \cdot 3 \cdot 5 \cdots(2 \ell-1)}{2 \cdot 4 \cdot 6 \cdots(2 \ell)}, \quad \ell \geq 1
$$

(with $\phi_{0}=1$ ), and where, using the digamma function $\Psi[1], \beta_{\ell}=\Psi(\ell+1)-\Psi(\ell+1 / 2)$.

In view of (2.9), the real part of the kernel may be expressed in the form

$$
g_{r}(\rho)=-\frac{1}{\pi} g_{r 1}(\rho) \ln \left(\frac{|\rho|}{R}\right)+g_{r 2}(\rho),
$$

where the functions $g_{r 1}$ and $g_{r 2}$ are given by

$$
g_{r 1}(\rho)=\frac{1}{\sqrt{\pi}} \sum_{n=0}^{\infty} \frac{(-1)^{n}(2 k a)^{2 n}}{(2 n) !} \Gamma(1 / 2-n)(|\rho| R)^{n} P_{-1 / 2}^{n}\left(2 \rho^{2}+1\right)
$$

and

$$
g_{r 2}(\rho)=\frac{1}{2 R} \sum_{n=0}^{\infty} \frac{(2 k a \rho)^{2 n}}{(2 n) !} F_{-1 / 2}^{n}\left(2 \rho^{2}+1\right) ;
$$

as shown in what follows, these functions are analytic for real values $|\rho|<\infty$.

The expression (2.13) for $g_{r 1}$ may be simplified considerably. To do this we use the integral representation

$$
P_{-1 / 2}^{n}\left(1+2 \rho^{2}\right)=\frac{1}{\pi^{3 / 2}}(-1)^{n} \Gamma(n+1 / 2)\left(\frac{|\rho|}{R}\right)^{n} \int_{0}^{\pi} \frac{\sin ^{2 n} \psi d \psi}{\sqrt{1+\rho^{2} \cos ^{2} \psi}},
$$

which follows from a corresponding integral representation for the hypergeometric function [14, eqn. IV(2)] together with a known expression [14, eqn. VI(45)] for the Legendre functions in terms of the hypergeometric function. Substituting equation (2.15) in (2.13) and reversing the order of summation and integration yields the simple expression

$$
g_{r 1}(\rho)=\frac{1}{\pi} \int_{0}^{\pi} \frac{\cos (2 k a \rho \sin \psi) d \psi}{\sqrt{1+\rho^{2} \cos ^{2} \psi}} .
$$


In addition to showing that $g_{r 1}(\rho)$ is, indeed, an analytic function for real values of $\rho$, this formula gives rise to an efficient method for the evaluation of this function in a neighborhood of the origin.

To show that $g_{r 2}$ (equation (2.14)) is analytic, in turn, we establish appropriate upper bounds on the growth with respect to $n$ of each one of the two sums in (2.10). To this end we first note from (2.11) that (i) $0<\phi_{\ell} \leq 1$ for $\ell=0,1,2, \ldots$, and (ii) $\phi_{\ell} \geq 1 /(2 \ell)$ for $\ell=1,2,3, \ldots$. Hence

$$
\frac{2}{\pi} \rho^{2 n} \sum_{m=1}^{n} \frac{1}{2 m} \frac{\phi_{n-m}}{\phi_{m}}\left(\frac{R}{\rho}\right)^{2 m} \leq \begin{cases}2 n R^{2 n} / \pi, & |\rho| \leq 1 \\ 2 n \rho^{2 n} R^{2 n} / \pi, & |\rho|>1 .\end{cases}
$$

In order to obtain a bound on the infinite series in (2.10), in turn, we recall [1] that the digamma function $\Psi$ is monotonically increasing for real $z>0$. It follows that $\Psi(z+1)-\Psi(z+1 / 2) \leq \Psi(z+1)-\Psi(z)=1 / z\left[1\right.$, eqn. 6.3.5], and thus $0<\beta_{m} \leq 1 / m$ for $m \geq 1$. Since $\phi_{m+n} \leq \phi_{m}$ and $\beta_{m+n} \leq \beta_{m}$ for all nonnegative integers $m$ and $n$, and since $\phi_{0}=1$ and $\beta_{0}=2 \ln 2$ [1, eqns. 6.3.2, 6.3.3], for all real $\rho$ we obtain the inequality

$$
\sum_{m=0}^{\infty}\left(\beta_{m}+\beta_{m+n}\right) \phi_{m} \phi_{m+n}\left(\frac{\rho}{R}\right)^{2 m} \leq 2 \sum_{m=0}^{\infty} \beta_{m} \phi_{m} \leq 4 \ln 2+2 \sum_{m=1}^{\infty} \frac{\phi_{m}}{m} .
$$

It is easy to check, further, that $0<\phi_{m}<C / m^{1 / 2}$ for some positive constant $C$, so that the series on the right-hand side of (2.18) is convergent, and thus the series on the left-hand side of (2.18), which is a positive quantity, is bounded above by a positive constant independent of $n$. (To check that $0<\phi_{m}<C / m^{1 / 2}$ for some constant $C$ note that $\ln \left(\phi_{m}\right)=\sum_{\ell=1}^{m} \ln (1-1 / 2 \ell)$, use the relation $\ln (1-x)=-x+\mathcal{O}\left(x^{2}\right)$, and, using an integral, estimate $\sum_{\ell=1}^{m} 1 / \ell \geq \ln (m)$.) The analyticity of $g_{r 2}(\rho)$ for all real $|\rho|<\infty$ then follows as a result of the rapid convergence of the series (2.14).

From the dimensional and dimensionless forms considered above for the kernel function, equations (1.5), (2.4), (2.1), and (2.12), we can write $F_{1}(z)=f_{1}(\rho) / a$ and $F_{2}(z)=f_{2}(\rho) / a$, where the dimensionless functions $f_{1}$ and $f_{2}$ are given by

$$
f_{1}(\rho) \equiv-\frac{1}{\pi} g_{r 1}(\rho) \quad \text { and } \quad f_{2}(\rho) \equiv \frac{1}{\pi} g_{r 1}(\rho) \ln (2 a R)+g_{r 2}(\rho)+i g_{i}(\rho) .
$$

In view of the rapid convergence of the series (2.7) it can be easily established that the function $g_{i}$ is analytic. Thus, the analyticity of $f_{1}$ and $f_{2}$, and therefore that of $F_{1}$ and $F_{2}$, follow from the analyticity results given above for $g_{r 1}$ and $g_{r 2}$.

2.3. Evaluation of the thin-wire kernel. As mentioned above, our algorithms for the solution of the Hallén and Pocklington problems rely on the new decomposition (1.5) of the integral kernel. More precisely, our algorithms require explicit, fast, and accurate evaluation of the analytic functions $F_{1}(z)$ and $F_{2}(z)$ for values of $z$ close to zero (to allow for explicit treatment to arbitrarily high order of the logarithmic singularity) as well as highly accurate evaluation of the full kernel $G(z)$ for values of $z$ away from the origin. These problems involve a degree of subtlety; the algorithms we devised for their solution are based on a number of representations for these functions and consideration of a number of subcases, as discussed in what follows.

Our numerical solvers require values of the kernel $G(z)=g(\rho) / a$ for all $z \in$ $[-2,2](\rho \in[-1 / a, 1 / a])$ and values of the functions $F_{1}(z)$ and $F_{2}(z)$ in subintervals of $[-1 / a, 1 / a]$ near the origin that are symmetric with respect to the origin. It is clear 
that, by symmetry, however, it suffices to evaluate the functions $F_{1}(z)$ and $F_{2}(z)$ and the kernel $G(z)$ for points $\rho \geq 0$. For accuracy and efficiency we use two different algorithms within this domain: one for $\rho \in[0, \Delta]$, near the kernel's singularity, and a different one for $\rho \in(\Delta, 1 / a]$, away from the singularity. The splitting parameter $\Delta$ is a suitably chosen $\mathcal{O}(1)$ constant; based on numerical experimentation we have selected the value $\Delta=5$, which was used in finding all of the numerical results presented in this paper. In some cases we use separate treatments for evaluation of the real and imaginary parts $g_{r}$ and $g_{i}$ of the kernel; see (2.4). Once these functions have been obtained, the corresponding dimensional forms in (1.5) may then be produced as indicated at the end of section 2.2 .

Evaluation of $g_{r}(\rho)$ and $g_{i}(\rho)$ in the region $\rho \in(\Delta, 1 / a]$. In this region our integral equation solver does not require separate evaluation of the functions $g_{r 1}(\rho)$ and $g_{r 2}(\rho)$. Both real and imaginary parts of the kernel are computed by means of the expansion

$$
\begin{aligned}
g(\rho)=\frac{\mathrm{e}^{i k a \tilde{R}}}{\tilde{R}}\left\{\sum_{n=0}^{M} \frac{(-1)^{n} \mathcal{A}_{n}\left[(k a)^{2}\right]}{2^{2 n}(n !)^{2} \tilde{R}^{2 n}}\right. \\
\left.\quad+i(k a) \sum_{n=1}^{M} \frac{(-1)^{n} n(2 n+1) \mathcal{B}_{n-1}\left[(k a)^{2}\right]}{2^{2 n}(n !)^{2} \tilde{R}^{2 n+1}}+\mathcal{O}\left(\frac{1}{\tilde{R}^{2 M+2}}\right)\right\}
\end{aligned}
$$

$\left(\tilde{R}=\sqrt{4 \rho^{2}+2}\right)$, which, as $M \rightarrow \infty$, converges to $g(\rho)$ for every real value of $\rho$. This expansion results as the finite-sum expression [1, eqn. 10.1.16] for the spherical Hankel function is substituted into Wang's expression (1.7) (thus avoiding evaluation of Hankel functions) and, subsequently, like powers of $\tilde{R}$ are collected. The coefficients $\mathcal{A}_{n}(x)$ and $\mathcal{B}_{n}(x)$ in (2.20) are polynomials of degree $n$; in Appendix A we present all of the polynomials necessary to evaluate the expansion (2.20) up to $M=10$. With $M=10$ this expansion yields at least double precision accuracy for all $k a \leq 1$ when $\rho \geq 3$ - certainly sufficient for our purposes, given our choice $\Delta=5$ of the splitting parameter.

Evaluation of $g_{i}(\rho)$ in the region $\rho \in[0, \Delta]$. In this domain the imaginary part of the kernel $g_{i}(\rho)$ is computed using the rapidly convergent expansion (2.7).

Evaluation of $g_{r}(\rho)$ in the region $\rho \in[0, \Delta]$. In the region $\rho \in[0, \Delta]$ our approach calls for separate evaluation of the analytic functions $g_{r 1}(\rho)$ and $g_{r 2}(\rho)$ of $(2.12)$ - to allow for explicit treatment to arbitrarily high order of the logarithmic singularity.

(a) Evaluation of $g_{r 1}(\rho)$ in the region $\rho \in[0, \Delta]$. Since the integrand in $(2.16)$ is analytic and periodic on the domain of integration, numerical evaluation of the integral by means of the trapezoidal rule gives rise to an exponentially convergent algorithm for evaluation of $g_{r 1}$. Naturally, for large values of $\rho$ this procedure would produce significant cancellation errors. For the limited set $[0, \Delta]$ of values of $\rho$ considered here, trapezoidal-rule integration with sufficiently many (rather few!) points yields full double precision accuracy. For efficiency, the function $g_{r 1}(\rho)$ is not computed by this method at every point where it is needed; instead this approach is used to precompute values of $f_{1}$ on a Chebyshev mesh within $[0, \Delta]$. These values are then used to produce a Chebyshev expansion $[8,12]$ of $g_{r 1}$-which can, in turn, be utilized to produce values of $g_{r 1}$ as needed.

(b) Evaluation of $g_{r 2}(\rho)$ in the region $\rho \in[0, \Delta]$. To evaluate the function $g_{r 2}(\rho)$ in this region, we introduce two subpartitions, $\rho \in[0, \delta]$ and $\rho \in(\delta, \Delta]$. The parameter $\delta$ is a suitably chosen small constant; based on our experiments we have selected the value $\delta=1 / 10$, which was used to find all of the numerical 
results presented in this paper. In the interval $(\delta, \Delta], g_{r 2}$ can be evaluated, quite simply, by means of the expressions

$$
g_{r 2}(\rho)=g_{r}(\rho)+\frac{1}{\pi} g_{r 1}(\rho) \ln \left(\frac{|\rho|}{R}\right)
$$

and

$$
g_{r}(\rho)=\frac{1}{2 \pi} \int_{0}^{\pi} \frac{\cos \left(2 k a \sqrt{\rho^{2}+\sin ^{2} \psi}\right) d \psi}{\sqrt{\rho^{2}+\sin ^{2} \psi}} .
$$

As in the case of the integral expression used for evaluation of the function $g_{r 1}$, the integrand in (2.22) is analytic (for real $\rho>0$ ) and periodic on the domain of integration, so that the trapezoidal rule converges exponentially fast. On the other hand, the computation of $g_{r 2}(\rho)$ in the interval $\rho \in[0, \delta]$ near the singular point is accomplished by direct summation of the series (2.14). The efficiency of this process is greatly improved by a simple observation: since both $P_{\nu}^{\mu}(z)$ and $Q_{\nu}^{\mu}(z)$ satisfy the recurrence relation [14, eqn. VI(25a)], it follows that so does the second term on the right-hand side of (2.9). It thus may be shown that the function $\Xi_{n}(\rho)=\rho^{2 n} F_{-1 / 2}^{n}\left(2 \rho^{2}+1\right)$ satisfies

$$
\Xi_{n+1}(\rho)=-\frac{2 n}{2 n+1}\left(1+2 \rho^{2}\right) \Xi_{n}(\rho)-\frac{2 n-1}{2 n+1} \rho^{2}\left(1+\rho^{2}\right) \Xi_{n-1}(\rho) .
$$

The necessary initial values $\Xi_{0}(\rho)$ and $\Xi_{1}(\rho)$ are obtained by the direct evaluation of (2.10), whose coefficients can themselves be generated recursively using the relations $\Gamma(z+1)=z \Gamma(z)$ and $\Psi(z+1)=\Psi(z)+1 / z[1]$. Using (2.23) the sequence of functions $\Xi_{n}(\rho)$ can be evaluated accurately and efficiently; clearly knowledge of these functions suffices to evaluate any truncation of the series on the right-hand side of (2.14). The function $g_{r 2}$ is precomputed on a Chebyshev mesh within $[0, \Delta]$ by the methods described in the present point (b); these values are then used to produce the Chebyshev expansion of $g_{r 2}$, to accompany the analogous expansion for $g_{r 1}$, discussed in point (a) above.

3. Regularity of solutions. It is well known that the solution $J$ of (1.1) and (1.3) with the end-point conditions (1.2) tends to zero like $\sqrt{1-z^{2}}$ as $z \rightarrow \pm 1[4,7,13]$. Jones [7] established that if $e(z)$ has a bounded derivative, then, under the conditions (1.2), equations (1.1) and (1.3) admit unique integrable solutions $J(z)$. Rynne [13] showed that an appropriate representation of the unknown current distribution is

$$
J(z)=\frac{I(z)}{\sqrt{1-z^{2}}}, \quad z \in[-1,1],
$$

where the function $I(z)$, which we refer to as the reduced current, is in the Sobolev space $W^{1, p}$ (with $p>1$ ), provided the right-hand side of the Hallén equation lies in $W^{2, p}$. The end-point conditions for $I(z)$, which result from the corresponding conditions (1.2) for $J(z)$, are given by

$$
I(-1)=I(1)=0 .
$$

Since, as established below, $I \in C^{1}[-1,1]$, provided the function $e$ on right-hand side of (1.1) is sufficiently smooth, it follows that the current $J$ vanishes at the end-points like a square root, as mentioned above. 
In this section, defining the reduced Hallén operator by

$$
\mathcal{H}\{I\}(z)=\int_{-1}^{1} \frac{G(z-t) I(t)}{\sqrt{1-t^{2}}} d t, \quad z \in[-1,1],
$$

we expand on the work of Rynne mentioned above and further relate the smoothness of a solution of the equation

$$
\mathcal{H}\{I\}=h
$$

to the smoothness of the right-hand side $h$. Our regularity proof is made possible by the kernel decompositions presented in section 2, together with a result concerning regularity of solutions of Symm's integral equation [15]. The actual result we use on Symm's equation, which is related to a theorem that can be found in [17], does not require an assumption made in that paper on the value of the logarithmic capacity of the interval of definition. Therefore, prior to the main discussion of this section on regularity of solutions of (3.4), which concludes with Theorem 3.7, we provide a brief discussion concerning Symm's equation.

Following [17], we call $C_{e}^{k}(2 \pi)$ (resp., $C_{e}^{\infty}(2 \pi)$ ) the set of all functions $v$ defined on the real line that are even, $2 \pi$-periodic, and $k$ times continuously differentiable (resp., infinitely differentiable). For a function $v \in C_{e}^{\infty}(2 \pi)$ and $s \geq 0$, further, we define the norm $\|\cdot\|_{s}$ by

$$
\|v\|_{s}^{2}=\left|a_{0}\right|^{2}+2 \sum_{m=1}^{\infty} m^{2 s}\left|a_{m}\right|^{2},
$$

where $a_{m}$ denotes the $m$ th cosine coefficient of $v$ :

$$
v(\theta)=\frac{1}{2} a_{0}+\sum_{m=1}^{\infty} a_{m} \cos (m \theta) .
$$

The Sobolev space $H_{e}^{s}(2 \pi)$ of even and $2 \pi$-periodic functions, finally, is defined as the completion of $C_{e}^{\infty}(2 \pi)$ with respect to the norm $\|\cdot\|_{s}$. The ordinary Sobolev spaces $H^{s}[a, b]$ will also be used in what follows (see, e.g., [2]), as will the spaces $C^{k}[a, b]$, $k=0,1, \ldots$ or $k=\infty$, of $k$-times continuously differentiable functions on the interval $[a, b]$.

The $2 \pi$-periodic even functions mentioned above arise in our context mainly through composition of various functions defined in the interval $[-1,1]$ with the function $\cos (\theta)$; since such compositions will occur frequently in what follows we introduce a special notation for them: for a function $g:[-1,1] \rightarrow \mathbb{R}$ we will write

$$
\tilde{g}(\theta)=g(\cos (\theta)) .
$$

Similarly, for an operator $\mathcal{K}$ whose codomain is a space of functions defined in the interval $[-1,1]$, we will denote by $\tilde{\mathcal{K}}$ the operator defined by

$$
\tilde{\mathcal{K}}\{I\}(\theta)=\mathcal{K}\{I\}(\cos (\theta)) .
$$

Finally, in what follows $[x]$ denotes the integer part of $x$. With these notations, we have the following lemma. 
LEMma 3.1. Let $s \geq 0$ and let $h_{0}$ be a measurable function defined in the interval $[-1,1]$ such that $\tilde{h}_{0} \in H_{e}^{s+1}(2 \pi)$ (where, per our conventions above, $\tilde{h}_{0}(\theta)=$ $\left.h_{0}(\cos (\theta))\right)$. If $I \in C^{0}[-1,1]$ satisfies Symm's integral equation

$$
h_{0}(z)=\int_{-1}^{1} \ln |z-t| \frac{I(t)}{\sqrt{1-t^{2}}} d t, \quad z \in[-1,1],
$$

then the following necessarily hold:

(a) $\tilde{I} \in H_{e}^{s}(2 \pi)$. In particular, if $h_{0} \in C^{\infty}[-1,1]$, then $\tilde{I} \in C_{e}^{\infty}(2 \pi)$.

(b) Let $k$ be a positive integer. Then for $s>k+1 / 2$ we further have $\tilde{I} \in$ $C^{k}(2 \pi)$ and $I \in C^{[k / 2]}[-1,1]$. In particular, for $h_{0} \in C^{\infty}[-1,1]$ we have $I \in C^{\infty}[-1,1]$.

Proof. The changes of variables $t=\cos \theta, z=\cos \alpha$ transform (3.7) into

$$
\tilde{h}_{0}(\alpha)=\int_{0}^{\pi} \ln |\cos \alpha-\cos \theta| \tilde{I}(\theta) d \theta, \quad \alpha \in \mathbb{R} .
$$

Since $\tilde{I} \in H_{e}^{0}(2 \pi)$ and $\tilde{h}_{0} \in H_{e}^{0}(2 \pi)$ we have the $H_{e}^{0}$-convergent cosine expansions

$$
\tilde{I}(\theta)=\frac{1}{2} b_{0}+\sum_{m=1}^{\infty} b_{m} \cos (m \theta)
$$

and

$$
\tilde{h}_{0}(\alpha)=\frac{1}{2} c_{0}+\sum_{m=1}^{\infty} c_{m} \cos (m \alpha)
$$

for these functions. As is well known, in view of (3.8) we have

$$
b_{m}=-\frac{1}{\pi} m c_{m} \quad \text { for } m \geq 1 ;
$$

see, e.g., $[11,17]$ or $\left[8\right.$, p. 211]. Thus, since $\tilde{h}_{0} \in H_{e}^{s+1}(2 \pi)$, we see that $\tilde{I} \in H_{e}^{s}(2 \pi)$ and point (a) follows. The fact that $\tilde{I} \in C^{k}(2 \pi)$ for $s>k+1 / 2$, on the other hand, follows directly from point (a) and Sobolev's lemma. Since $\tilde{I}$ is even and $2 \pi$ periodic, finally, it is easy to check that $\tilde{I} \in C^{k}(2 \pi)$ implies that $I(t)=\tilde{I}\left(\cos ^{-1}(t)\right)$ is a $[k / 2]$-times continuously differentiable function, and the proof of the lemma is thus complete.

REMARK 3.2. The function $\tilde{h}_{0}$ may be significantly smoother than $h_{0}$ (take, for example, $h_{0}(z)=\sqrt{1-z^{2}}$, for which we have $\tilde{h}_{0} \in H_{e}^{1}(2 \pi)$ but $h_{0} \notin H_{e}^{1}(2 \pi)$, and for which $\tilde{h}_{0}$ has a bounded first derivative while $h_{0}$ does not). This observation has important consequences concerning the applicability of the results of this section; see Remark 3.8 below.

Continuing with the preliminaries for our main proof, we define the operator

$$
\mathcal{T}\{I\}(z)=\int_{-1}^{1} \frac{F_{1}(z-t) I(t)}{\sqrt{1-t^{2}}} \ln |z-t| d t=\mathcal{H}\{I\}(z)-\int_{-1}^{1} \frac{F_{2}(z-t) I(t)}{\sqrt{1-t^{2}}} d t
$$

where $F_{1}$ and $F_{2}$ are the analytic functions in (1.5), and where $z$, as in many analogous situations throughout this section, lies in the interval $[-1,1]$; to simplify the notation, the statement $z \in[-1,1]$ is henceforth suppressed when its validity is clear from the context. Since $F_{2}$ is analytic (section 2.2 ) and since $J=I / \sqrt{1-z^{2}}$ is integrable it 
follows that the last integral in (3.9) is infinitely differentiable. Thus, taking into account our conventions (3.5)-(3.6), we note the following.

REMARK 3.3. If (3.4) is satisfied with $\tilde{h} \in H_{e}^{s+1}(2 \pi)$, then $\tilde{\mathcal{T}}\{I\} \in H_{e}^{s+1}(2 \pi)$.

To establish our regularity result for $I(z)$, let

$$
F_{1,2 m}(z)=\sum_{k=0}^{m} \frac{F_{1}^{(2 k)}(0)}{(2 k) !} z^{2 k}
$$

denote the truncated Taylor series of $F_{1}$ (it is easy to verify from (2.16) and (2.19) that all of the odd derivatives of $F_{1}$ vanish at $z=0$ ). Further, define the operators

$$
\mathcal{S}_{2 m}\{I\}(z)=\int_{-1}^{1} \frac{F_{1,2 m}(z-t) I(t)}{\sqrt{1-t^{2}}} \ln |z-t| d t
$$

and

$$
\mathcal{R}_{2 m}\{I\}(z)=\int_{-1}^{1} \frac{\left[F_{1}(z-t)-F_{1,2 m}(z-t)\right] I(t)}{\sqrt{1-t^{2}}} \ln |z-t| d t
$$

so that

$$
\mathcal{T}\{I\}=\mathcal{S}_{2 m}\{I\}+\mathcal{R}_{2 m}\{I\} .
$$

For all nonnegative integers $m, \mathcal{R}_{2 m}\{I\} \in C^{2 m+2}[-1,1]$, so that in view of Remark 3.3 we have the following.

Remark 3.4. Assume (3.4) is satisfied with $\tilde{h} \in H_{e}^{s+1}(2 \pi)$. Then defining, for fixed $s, p_{m}=\min (s+1,2 m+2)$, for all nonnegative integers $m$ we have $\tilde{\mathcal{S}}_{2 m}\{I\} \in$ $H_{e}^{p_{m}}(2 \pi)$.

In view of (3.10) we have

$$
\mathcal{S}_{2 m}\{I\}=\sum_{k=0}^{m} \frac{F_{1}^{(2 k)}(0)}{(2 k) !} \mathcal{V}_{2 k}\{I\},
$$

where

$$
\mathcal{V}_{\ell}\{I\}(z)=\int_{-1}^{1} \frac{(z-t)^{\ell} I(t)}{\sqrt{1-t^{2}}} \ln |z-t| d t
$$

The following lemma follows directly by differentiation of (3.15).

Lemma 3.5. Suppose $I \in C^{0}[-1,1]$. Then, for every integer $k \geq 0, \mathcal{V}_{k}\{I\} \in$ $C^{k}[-1,1]$ and

$$
\mathcal{V}_{k}^{(k)}\{I\}=k !\left(\mathcal{V}_{0}\{I\}-\mathcal{W}_{k}\{I\}\right),
$$

where $\mathcal{W}_{k}\{I\}$ is a constant given by

$$
\mathcal{W}_{k}\{I\}=\left[\sum_{m=1}^{k}\left(\begin{array}{c}
k \\
m
\end{array}\right) \frac{(-1)^{m}}{m}\right] \cdot \int_{-1}^{1} \frac{I(t)}{\sqrt{1-t^{2}}} d t .
$$

The connection between Symm's integral equation and Hallén's integral equation stems from the (inductive) proof of the following lemma. (Note that $\mathcal{V}_{0}$ coincides with the Symm's integral operator on the right-hand side of (3.7).) 
Lemma 3.6. Let $s \geq 0$ and assume (3.4) is satisfied with $\tilde{h} \in H_{e}^{s+1}(2 \pi)$. Then $\tilde{\mathcal{V}}_{0}\{I\} \in H_{e}^{s+1}(2 \pi)$.

Proof. By induction in $m=0,1 \ldots$ we establish that $\tilde{\mathcal{V}}_{0}\{I\} \in H_{e}^{p_{m}}(2 \pi)$ for all integers $m$, where $p_{m}=\min (s+1,2 m+2)$ (see Remark 3.4). Taking $m$ such that $2 m+2 \geq s+1$ then shows that $\tilde{\mathcal{V}}_{0}\{I\} \in H_{e}^{s+1}(2 \pi)$, as desired.

For $m=0$, we note from (3.14) that

$$
\mathcal{S}_{0}\{I\}=F_{1}(0) \mathcal{V}_{0}\{I\} .
$$

Since $F_{1}(0) \neq 0$ (see $(2.1),(2.16)$, and $\left.(2.19)\right)$ and since, by Remark 3.4, $\tilde{\mathcal{S}}_{0}\{I\} \in$ $H_{e}^{p_{0}}(2 \pi)$, it follows that $\tilde{\mathcal{V}}_{0}\{I\} \in H_{e}^{p_{0}}(2 \pi)$, as needed.

Assume now that $\tilde{\mathcal{V}}_{0}\{I\} \in H_{e}^{p_{m}}(2 \pi)$. In the case $2 m+2 \geq s+1$ we have $p_{m+1}=p_{m}$ and the inductive step is complete. Let us consider, then, the case $2 m+2<s+1$. We know that $\tilde{\mathcal{V}}_{0}\{I\} \in H_{e}^{p_{m}}(2 \pi)$ and thus, from Lemma 3.5, that $\tilde{\mathcal{V}}_{2 k}\{I\} \in H_{e}^{p_{m}+2 k}(2 \pi)$. In particular, for all $k \geq 1, \tilde{\mathcal{V}}_{2 k}\{I\} \in H_{e}^{p_{m}+2}(2 \pi)$. In view of the relation (3.14) we have

$$
\tilde{\mathcal{S}}_{2 m+2}\{I\}=\sum_{k=0}^{m+1} \frac{F_{1}^{(2 k)}(0)}{(2 k) !} \tilde{\mathcal{V}}_{2 k}\{I\} .
$$

Since as noted above $F_{1}(0) \neq 0$, and since by Remark $3.4 \tilde{S}_{2 m+2} \in H_{e}^{p_{m+1}}(2 \pi)$, it follows that $\tilde{\mathcal{V}}_{0}\{I\} \in H_{e}^{r}(2 \pi)$, where $r=\min \left\{p_{m+1}, p_{m}+2\right\}=p_{m+1}$, and thus the inductive step and the proof of the lemma are complete.

In view of Lemmas 3.1, 3.6, and the Sobolev lemma, the main result of this section now follows directly.

Theorem 3.7. Assume I satisfies (3.4) with $\tilde{h} \in H_{e}^{s+1}(2 \pi)$. Then $\tilde{I} \in H_{e}^{s}(2 \pi)$. Further, if $h \in C^{k}[-1,1]$, then $\tilde{I} \in C_{e}^{k-2}(2 \pi)$ and $I \in C^{[(k-2) / 2)]}[-1,1]$. In particular, if $h \in C^{\infty}[-1,1]$, then $\tilde{I} \in C^{\infty}[0, \pi]$ and $I \in C^{\infty}[-1,1]$.

REMARK 3.8. This theorem extends Rynne's $s=1$ regularity result (Theorem 4.2 in [13]) to all $s \geq 0$, and thus, in particular, it allows us to show for the first time that if the right-hand side $h$ of the Hallén equation is infinitely differentiable, then so is its solution I. In the particular case $s=1$ we note that Theorem 3.7, while similar to Rynne's, is not identical to it: Rynne's theorem is in some ways stronger and in other ways weaker than the $s=1$ version of ours. Indeed, Rynne's result establishes that the reduced current I lies in the Sobolev space $W^{1, p}(p>1)$, provided the right-hand side $h$ lies in $W^{2, p}$. Considering the particular value $p=2$ and with reference to Remark 3.2 , we indeed see that our $s=1$ hypothesis, namely, $\tilde{h} \in H_{e}^{2}(2 \pi)$, is less stringent than that in Rynne's result, and thus the present result is more generally applicable. For $h \in H^{2}[-1,1]=W^{2,2}[-1,1]$ the conclusion in Rynne's result is stronger than our $s=1$ result, however, since it shows that not only $\tilde{I}$ but also $I$ is in an $H^{1}$ function.

4. Numerical treatment of the Hallén and Pocklington equations. In this section we present our solvers for the Hallén and Pocklington problems. The theoretical equivalence of the Hallén and Pocklington equations is well established [7, 13]; a comparison of the performance of our numerical solvers for these equations is provided in section 5. In section 4.1 we introduce our Hallén solver, which we base on a Chebyshev series representation of the reduced current function (equation (3.1)), the use of which allows us to incorporate explicitly the current's end-point singularities within a high-order treatment. Our Pocklington solver, which results as a natural extension of the methods of section 4.1, is then presented in section 4.2. Finally, in section 4.3, we introduce an alternative formulation of the Hallén problem, which is suitable for use in problems involving very thin wires. 
4.1. Hallén solver. Since the singular weight function $\left(1-t^{2}\right)^{-1 / 2}$ in the integrand of (3.3) can be eliminated by means of the Chebyshev change of variables $t=\cos \theta$ [8, eqn. (1.1)], the set of Chebyshev polynomials $T_{n}(z)$ is a natural choice of basis functions to represent $I(z)$ for $z \in[-1,1]$. We thus use the discretization

$$
I(z) \approx \frac{1}{2} b_{0}+\sum_{n=1}^{N} b_{n} T_{n}(z),
$$

where the Chebyshev coefficients $b_{n}$ are the unknowns of the problem. The use of such an expansion is highly advantageous: for the case of an incident plane wave, for example, the solution $I \in C^{\infty}[-1,1]$ (see section 3), and the Chebyshev series (4.1) thus converges faster than $\mathcal{O}\left(N^{-m}\right)$ for any positive integer $m$-i.e., it achieves superalgebraic convergence.

From (4.1), the action of the Hallén operator $\mathcal{H}$ in (3.3) can be approximated by

$$
\mathcal{H}\{I\}(z) \approx \frac{1}{2} b_{0} A_{0}(z)+\sum_{n=1}^{N} b_{n} A_{n}(z)
$$

where $A_{n}=\mathcal{H}\left\{T_{n}\right\}$. In Appendix B we tackle the difficult and important problem of evaluating the functions $A_{n}$ accurately and efficiently. The problems of evaluating both the kernel of the operator $\mathcal{H}$ (section 2.3) and the functions $A_{n}$, which present similar challenges, constitute two of the main contributions of this paper.

The discrete form (4.2) of the operator $\mathcal{H}$ is the basis of our collocation method to solve the Hallén problem. Each column of the resulting system matrix corresponds to a particular index $n$ of the function $A_{n}$, while the rows are generated by testing (4.2) at $N+1$ values of $z \in[-1,1]$.

REMARK 4.1. During the course of this study, it was observed that the condition number of the system matrix is strongly dependent on the selection of the collocation points: we have found that the selection of a uniformly spaced set results in very poor conditioning, while testing at the set of Chebyshev points

$$
z_{j}=\cos \left(\frac{j \pi}{N}\right), \quad j=0,1,2, \ldots, N,
$$

results in well-conditioned linear systems; see section 5.

Jones [7] has shown that the solution to the Hallén problem may be obtained by linear superposition. We implement Jones's idea in our context by writing the reduced current as the sum

$$
I(z)=\alpha_{1} I^{(1)}(z)+\alpha_{2} I^{(2)}(z)+I^{(3)}(z),
$$

where $\mathcal{H}\left\{I^{(1)}\right\}(z)=\cos k z, \mathcal{H}\left\{I^{(2)}\right\}(z)=\sin k z$, and

$$
\mathcal{H}\left\{I^{(3)}\right\}(z)=-4 \pi i \int_{-1}^{z} e(t) \sin [k(z-t)] d t
$$

the values of the constants $\alpha_{1}$ and $\alpha_{2}$ follow from the end-point conditions (3.2). Since, as is easy to check, we have $I^{(1)}(1)=I^{(1)}(-1)$ and $I^{(2)}(1)=-I^{(2)}(-1)$, it follows that

$$
\alpha_{1}=-\frac{1}{2}\left(\frac{I^{(3)}(1)+I^{(3)}(-1)}{I^{(1)}(1)}\right) \quad \text { and } \quad \alpha_{2}=-\frac{1}{2}\left(\frac{I^{(3)}(1)-I^{(3)}(-1)}{I^{(2)}(1)}\right) .
$$


To obtain $I$ numerically we use a representation of the form (4.1) for each one of the quantities $I^{(1)}, I^{(2)}$, and $I^{(3)}$. The corresponding Chebyshev coefficients $b_{n}^{(j)}$ of $I^{(j)}(z)(n=0,1, \ldots)$ are obtained from the equations for the $I^{(j)}$ 's (equation $(4.5)$ and immediately above) and repeated use of the LU decomposition of the $(N+1) \times(N+1)$ matrix that discretizes the operator $\mathcal{H}$ (as described earlier in this section), together with back- and forward substitution for $j=1,2,3$. Once these Chebyshev coefficients have been obtained, the constants $\alpha_{1}$ and $\alpha_{2}$ are calculated by means of (4.6), using the Chebyshev series to produce $I^{(j)}( \pm 1)$. The reduced current $I$ is then found by summation of its Chebyshev series, whose coefficients are given by

$$
b_{n}=\alpha_{1} b_{n}^{(1)}+\alpha_{2} b_{n}^{(2)}+b_{n}^{(3)}, \quad n=0,1, \ldots, N .
$$

4.2. Pocklington solver. A method for solving the Pocklington problem embodied in (1.1) and (1.2), which uses, again, a representation of the form (4.1) and the coefficients $b_{n}$ as unknowns, is readily derived from results presented in section 4.1. To obtain such a method we first represent the functions $A_{n}(z)$ by truncated Chebyshev expansions

$$
A_{n}(z) \approx \frac{1}{2} a_{0}^{(n)}+\sum_{m=1}^{N} a_{m}^{(n)} T_{m}(z)
$$

The coefficients in the series (4.8) can be obtained directly from an (FFT-based) Chebyshev transform of the columns of the matrix for the Hallén problem-which, by construction, contain the point values of the functions $A_{n}$ at the Chebyshev points (4.3).

Using the coefficients in (4.8) we can then obtain the Chebyshev series coefficients of $\left(\partial^{2} / \partial z^{2}+k^{2}\right) A_{n}(z)$

$c_{m}^{(n)}=\frac{2}{\pi} \int_{-1}^{1} \frac{T_{m}(z)}{\sqrt{1-z^{2}}}\left(\frac{\partial^{2}}{\partial z^{2}}+k^{2}\right) A_{n}(z) d z=k^{2} a_{m}^{(n)}+4 \sum_{\ell=1}^{\left[\frac{N-m}{2}\right]} \ell(\ell+m)(2 \ell+m) a_{2 \ell+m}^{(n)}$,

which is valid for $m=0,1,2, \ldots$. The second derivative of the Chebyshev polynomial in (4.9) is evaluated by means of the relation [8, p. 38].

The Pocklington system matrix contains the quantity $c_{m}^{(n)}$ in the $m$ th row and $n$th column. Since it is necessary to enforce vanishing of the reduced current at the end-points $z= \pm 1$ we only use the first $N-1$ of these equations to allow for the two additional equations enforcing the end-point conditions (3.2):

$$
\frac{1}{2} b_{0}+\sum_{n=1}^{N} b_{n}=0 \quad \text { and } \quad \frac{1}{2} b_{0}+\sum_{n=1}^{N}(-1)^{n} b_{n}=0 .
$$

The right-hand side of the system consists of the first $N-1$ Chebyshev coefficients of $-4 \pi i k e(z)$ and zeros in the last two positions. The system is solved directly by an LU decomposition.

4.3. Alternative Hallén method for very thin wires. For particular physical cases involving very thin wires $(a \ll$ wire-length, independently of the value of $\mathrm{ka}$ ), the rate of convergence to the solution may be substantially improved by means of a distribution of discretization points within the wire different from those used 
in the previous sections. We speculate that the slower convergence in the absence of such change of variables must be the result of a small-weight singularity in the thin-wire solutions located near the origin on the imaginary axis (associated with a corresponding singularity, the kernel itself has at $\left(z-z^{\prime}\right)=2 a i$; see (1.4)). While the methods outlined in sections 4.1 and 4.2 exhibit superalgebraic convergence even for such cases, use of the point-distributions described in what follows, which are more concentrated on the end-points than those considered in previous sections, result in significant additional convergence acceleration. For the sake of brevity, here we restrict attention to the Hallén equation; the development of this method proceeds in analogy to that presented in section 4.1.

In contrast with the Chebyshev (cosine) change of variables used earlier in this text, here we introduce the coordinate transformation

$$
z=\frac{2 \cos w}{1+\cos ^{2} w}
$$

which maps $w \in[0, \pi]$ to $z \in[-1,1]$ and which, like the cosine change of variables used earlier, eliminates the end-point singularities in the integrand of (3.3). The cosine change of variables results in a locally quadratic clustering of points near the ends of the wire; the clustering of points near the ends of the wire induced by the transformation (4.11), on the other hand, is quartic. It is precisely this property which gives rise to the improved convergence rates of the solution for very thin wires. The reduced current is an even, periodic function of the new variables, and thus it may be approximated by the cosine series

$$
I\left(\frac{2 \cos w}{1+\cos ^{2} w}\right) \approx \frac{1}{2} b_{0}+\sum_{n=1}^{N} b_{n} \cos (n w),
$$

where the coefficients $b_{n}$ are the unknowns of the problem. For $I(z) \in C^{m+1}[-1,1]$, the error of the cosine series (4.12) is $\mathcal{O}\left(N^{-m}\right)$ for all $w \in[-\pi, \pi]$. In particular, for $I(z) \in C^{\infty}[-1,1]$, the cosine series (4.12) converges superalgebraically to $I(z)$. As discussed in section 5.1, for thin wires, the representation (4.12) converges much more quickly than the expansion (4.1).

In the new variables, the Hallén operator may be approximated by the discrete form

$$
\mathcal{H}\{I\}\left(\frac{2 \cos w}{1+\cos ^{2} w}\right) \approx \frac{1}{2} b_{0} B_{0}(w)+\sum_{n=1}^{N} b_{n} B_{n}(w) .
$$

The integral functions resulting from the method may be written as

$$
B_{n}(w)=-2 \int_{0}^{\pi} \frac{\sin u \cos (n u)}{1+\cos ^{2} u} G\left(\frac{2 \cos w}{1+\cos ^{2} w}-\frac{2 \cos u}{1+\cos ^{2} u}\right) d u .
$$

Much in the same way as in section 4.1, the discrete form of the operator (4.13) is the basis for a collocation method to solve the Hallén problem. The collocation points are chosen to be the $N+1$ uniformly spaced values of $w \in[0, \pi]$, which, as will be demonstrated in section 5 , results in a well-conditioned system. The total current is found by our adaptation of Jones's method, as outlined in (4.4) and below. 
TABLE 5.1

Correspondence of parameters between [4] and the present text. The wire occupies the interval $[0,1]$ in that work, while it occupies the interval $[-1,1]$ here.

\begin{tabular}{cc}
\hline Present & Reference [4] \\
\hline$a$ & $2 a$ \\
$k$ & $\omega / 2$ \\
$k a$ & $\omega a$ \\
\hline
\end{tabular}

5. Results and discussion. In addition to several efficient novel algorithms for wire problems, the contribution [4] presents an excellent survey of modern methods in the area; we therefore use the results in that reference to demonstrate the improvements that can be offered by our algorithms. We note that only the Pocklington equation is treated in [4], and, further, that our methods differ substantially from those presented in that contribution. In fact, several methods are discussed in [4], some which exhibit $\mathcal{O}\left(N^{-1}\right)$ or $\mathcal{O}\left(N^{-2}\right)$ convergence in the solution, and some methods which exhibit much faster convergence. In particular, the hp-geometric Galerkin scheme introduced in [4] appears to exhibit superalgebraic convergence and is, quite definitely, the most efficient and accurate of the methods available previously. In what follows, we therefore compare our results to hp-geometric Galerkin data in every instance for which such data was made available in [4]; our results compare much more favorably to all others in the literature.

The identification of parameters and unknowns between [4] and the present text is as described in Table 5.1. In that reference, the right-hand side of the Pocklington equation is taken to equal unity in all cases. To account for this prescription, as well as the different kernel normalization and wire-length, we set $\mathcal{H}\left\{I^{(3)}(z)\right\}=\pi / k^{2}$ for the Hallén problem, and $e(z)=i /(4 k)$ for the Pocklington problem; with these definitions the current $J$ we consider matches that of [4].

In this section the maximum relative errors $e_{\max }$ are defined by

$$
e_{\max }=\frac{\max _{j}\left|J\left(z_{j}\right)-J_{r e f}\left(z_{j}\right)\right|}{\max _{j}\left|J_{r e f}\left(z_{j}\right)\right|} .
$$

The reference data sets $J_{r e f}\left(z_{j}\right)$ we used for evaluation of $e_{\max }$ were obtained using large numbers $N+1$ of unknowns, to ensure high accuracies. The numerical values of $e_{\max }$ displayed in the tables, in turn, were obtained by evaluating $J$ and $J_{\text {ref }}$ at sufficiently fine Chebyshev meshes $\left\{z_{j}\right\}$, so that the quantity $\left|J(z)-J_{\text {ref }}(z)\right|$, which experiences rapid oscillations near the wire end-points, is fully resolved, and thus allows for accurate computation of $e_{\max }$. See the captions of Tables 5.2 to 5.6 for full details on the meshes used for evaluation of $J_{\text {ref }}$ and $e_{\max }$. Details concerning the accurate evaluation of the total current $J(z)=I(z) / \sqrt{1-z^{2}}$ from the reduced current $I(z)$ are presented in Appendix C; here we simply point out that a straightforward division does give rise to significant error increases - unbounded, in fact, as the evaluation points tend toward the end-points. The strategies described in Appendix C completely resolve this difficulty and result in currents $J$ that are as accurate as the corresponding reduced currents $I$.

In what follows we first examine the convergence rates of our algorithms, with attention to the relative advantages offered by our Hallén, Pocklington, or alternative Hallén approaches. All computations presented here were performed on an AMD 2600+ computer system. Solutions of all linear systems were obtained by means of the LU-based direct solvers provided in the LAPACK linear algebra package 

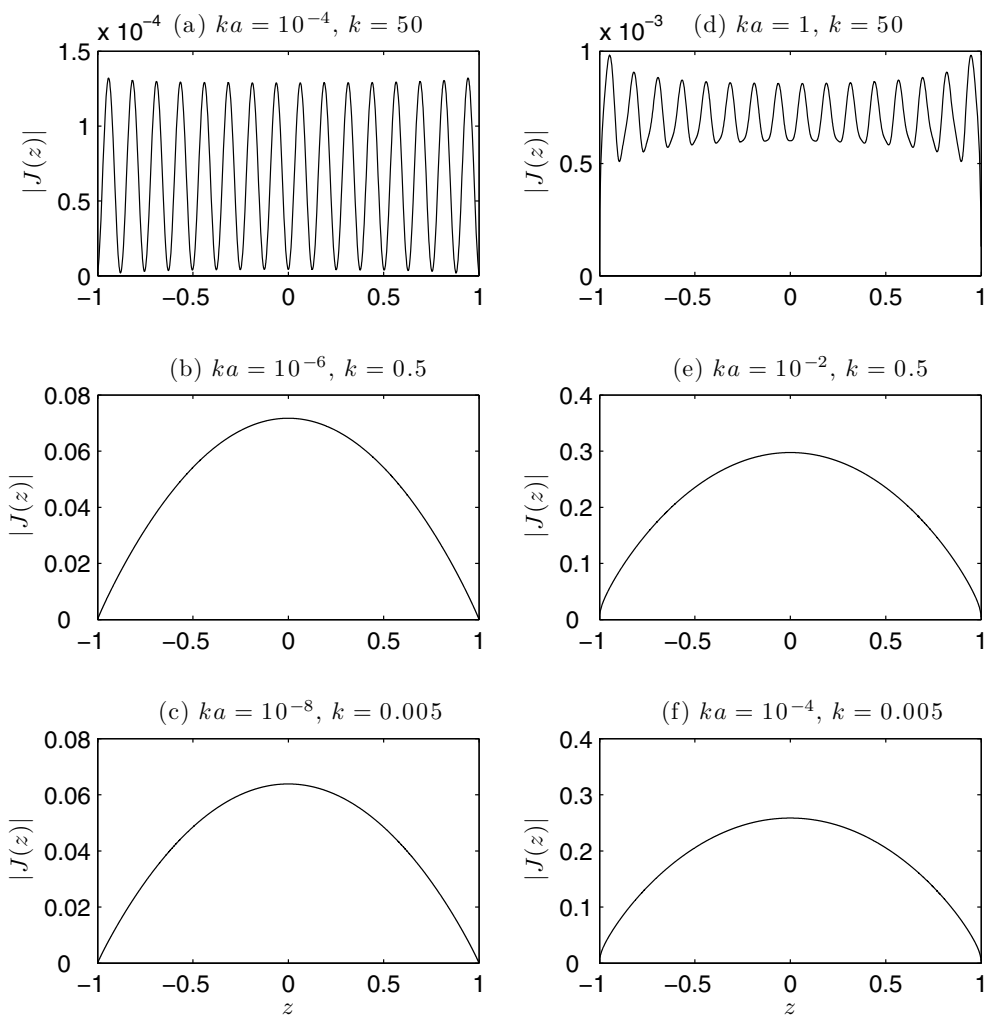

Fig. 5.1. Profiles of the current function $J(z)$ studied by Davies, Duncan, and Funken [4].

(www.netlib.org/lapack/). In a rather comprehensive set of tests that we consider, four-digit solutions, for example, were found in a execution time of at most four seconds; in most cases computing time for a four-digit solve was a fraction of a second. As shown below, further, in all cases much higher accuracies result in very small additional computing times: see Tables 5.2 to 5.6 in section 5.1; in all tables the execution time is denoted by $T$.

5.1. Numerical results. To demonstrate the superalgebraic convergence of our methods we consider a set of six test cases put forward in [4], which span a broad range of physical configurations. The profiles of the total currents $J$ for these cases are displayed in Figure 5.1; note that all cases in the left-hand column of that figure have radii $a=0.5 \cdot 10^{-6}$, while those in the right-hand column have radii $a=0.5 \cdot 10^{-2}$. The tables presented in this section display, among other quantities, the computational times required to obtain the Chebyshev series of the reduced current $I$; in all cases the table captions list the (very small) corresponding additional times required to evaluate the current $J$ on a fine Chebyshev grid (of a size also listed on the table captions); see also Appendix C. Note, however, that in the context of our algorithm, it is possible (and most efficient) to use the reduced current $I$, not the physical current $J$, to evaluate the scattered fields in space. Thus, if the quantity of interest is the field scattered by the wire, the calculation of the current $J$ is unnecessary. 
TABLE 5.2

Alternative Hallén solver for case (a): $k=50$ and $k a=10^{-4}$ (very thin wires). The current profile is shown in Figure 5.1(a). An N+1-term cosine series is used, and the algorithm is run with integration parameters $M_{1}$ and $M_{2}$ and splitting parameter $\Delta=5$ (see Appendix $\mathrm{C}$ ). The quantity $C_{N}$ is the estimate of the reciprocal condition number provided by the linear algebra package LAPACK. The error oscillations are fully resolved: the maximum relative error $e_{\max }$ (see (5.1)) was obtained by evaluation of $J$ and $J_{\text {ref }}$ on a $2^{13}+1$-point Chebyshev grid; the reference solution $J_{\text {ref }}$ was obtained with $N=240, M_{1}=2^{12}$, and $M_{2}=2^{9}$. The evaluation of $J$ at the $2^{13}+1$-point Chebyshev grid required approximately $0.07 \mathrm{~s}$ of computational time in addition to the tabulated computing times. Note from Figure 5.1(a) that the maximum value of the solution is of the order of $10^{-4}$ so that the maximum absolute errors decrease up to order of $10^{-12}$-consistent, for the 16-digit accuracy used, with a condition number of the order of $10^{-4}$ listed above.

\begin{tabular}{cccccc}
\hline$N$ & $M_{1}$ & $M_{2}$ & $T(\mathrm{~s})$ & $e_{\max }$ & $C_{N}$ \\
\hline 110 & $2^{7}$ & $2^{2}$ & 0.28 & $3.3 \cdot 10^{-1}$ & $7.8 \cdot 10^{-4}$ \\
120 & $2^{8}$ & $2^{2}$ & 0.63 & $2.1 \cdot 10^{-2}$ & $5.8 \cdot 10^{-4}$ \\
130 & $2^{9}$ & $2^{3}$ & 1.50 & $2.0 \cdot 10^{-3}$ & $4.2 \cdot 10^{-4}$ \\
140 & $2^{10}$ & $2^{3}$ & 3.43 & $6.1 \cdot 10^{-5}$ & $3.8 \cdot 10^{-4}$ \\
150 & $2^{11}$ & $2^{4}$ & 8.11 & $3.2 \cdot 10^{-6}$ & $3.6 \cdot 10^{-4}$ \\
160 & $2^{11}$ & $2^{4}$ & 9.18 & $7.3 \cdot 10^{-8}$ & $3.4 \cdot 10^{-4}$ \\
170 & $2^{11}$ & $2^{4}$ & 10.29 & $2.0 \cdot 10^{-8}$ & $3.2 \cdot 10^{-4}$
\end{tabular}

Case (a) $k a=10^{-4}$ and $k=50$. The current profile is shown in Figure 5.1(a). The relative error and the reciprocal matrix condition number (estimated by LAPACK) are presented in Table 5.2 for increasing values of the index $N$ of truncation of the solution's cosine expansion. For this case, a very thin wire problem, we used the alternative Hallén method described in section 4.3. We see from Table 5.2 that, for example, full single precision accuracy results from this method in an $N=160$, 9-second computation. (Use of the regular Hallén and Pocklington methods of sections 4.1 and 4.2 requires much larger numbers of unknowns, of the order of $N=800$, to achieve comparable accuracies.) Note that the condition number remains virtually constant throughout the table. A graphical solution $J$, consistent with our Figure 5.1 (a), was given in [4] (case $a=1 e-6, \omega=100$ in that work), but errors were not reported there for this case. Through comparison with cases (c) and (d) below we speculate that the hp-geometric Galerkin method of [4] in the present case (a) requires a number of the order of at least $N=350$ unknowns to achieve errors of the order of $10^{-5}$ - since, in the present case, the discretization needs to resolve both the extreme end-point singularities arising from the very thin wire (for which hp-geometric Galerkin requires $N=50$ in case (c)) as well as the higher frequency $k=50$ (which for hp-geometric Galerkin requires $N=350$ in case (d)). Taking into account, further, the superalgebraic convergence (in $M_{1}$ and $M_{2}$ ) of the integration methods we use versus the third-order or lower convergence inherent in previous methods (see paragraph (b) in the introduction), we expect the approaches in the present paper should prove significantly advantageous over most solvers otherwise available.

Case (b) $k a=10^{-6}$ and $k=0.5$. The current profile is shown in Figure 5.1(b). Statistics for this case are virtually identical to those of case (c). A graphical solution $J$, consistent with our Figure 5.1(b), was given in [4] (case $a=1 e-6, \omega=1$ in that work). In that reference, errors were reported for slower numerical methods (which require approximately 100 unknowns to produce errors of order $10^{-3}$ ), but not for the hp-geometric Galerkin approach, which is the main subject of our comparison.

Case (c) $k a=10^{-8}$ and $k=0.005$. The current profile is shown in Figure $5.1(\mathrm{c})$. The relative error and the reciprocal matrix condition number are presented 
TABLE 5.3

Same as Table 5.2, with the following exceptions: $k=0.005, k a=10^{-8}$; the current profile is shown in Figure 5.1(c); the reference solution $J_{\text {ref }}$ was obtained with $N=140, M_{1}=2^{12}$, and $M_{2}=2^{9}$; in this case, in which the maximum value of the solution is of order 0.08, the condition number is not consistent with stagnation at the level $10^{-8}$ - which, in fact, does not occur: a certain oscillatory behavior exhibited by the cosine series coefficients, shown in Figure 5.2, gives rise to nonmonotone convergence.

\begin{tabular}{cccccc}
\hline$N$ & $M_{1}$ & $M_{2}$ & $T(\mathrm{~s})$ & $e_{\max }$ & $C_{N}$ \\
\hline 10 & $2^{9}$ & $2^{2}$ & 0.02 & $1.9 \cdot 10^{-1}$ & $4.4 \cdot 10^{-3}$ \\
20 & $2^{10}$ & $2^{2}$ & 0.11 & $2.4 \cdot 10^{-4}$ & $2.5 \cdot 10^{-3}$ \\
30 & $2^{11}$ & $2^{3}$ & 0.41 & $4.5 \cdot 10^{-6}$ & $2.0 \cdot 10^{-3}$ \\
40 & $2^{11}$ & $2^{4}$ & 0.69 & $5.2 \cdot 10^{-7}$ & $1.3 \cdot 10^{-3}$ \\
50 & $2^{11}$ & $2^{4}$ & 1.02 & $5.2 \cdot 10^{-7}$ & $1.1 \cdot 10^{-3}$ \\
60 & $2^{11}$ & $2^{4}$ & 1.41 & $3.5 \cdot 10^{-7}$ & $9.0 \cdot 10^{-4}$ \\
70 & $2^{11}$ & $2^{4}$ & 1.87 & $2.5 \cdot 10^{-7}$ & $7.9 \cdot 10^{-4}$ \\
80 & $2^{11}$ & $2^{4}$ & 2.44 & $9.8 \cdot 10^{-8}$ & $6.9 \cdot 10^{-4}$ \\
90 & $2^{11}$ & $2^{4}$ & 3.02 & $7.4 \cdot 10^{-8}$ & $6.0 \cdot 10^{-4}$ \\
100 & $2^{11}$ & $2^{4}$ & 3.64 & $3.1 \cdot 10^{-8}$ & $5.4 \cdot 10^{-4}$ \\
\hline
\end{tabular}

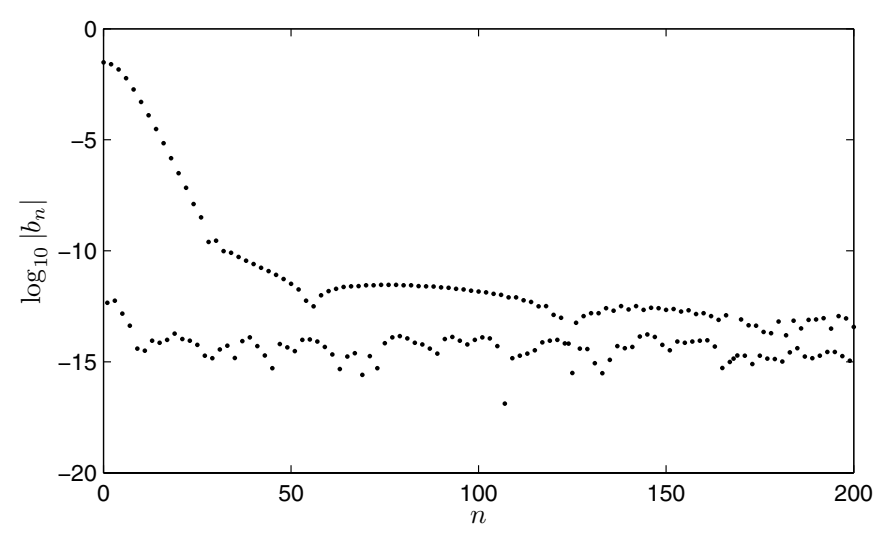

FIG. 5.2. Cosine series coefficients of the alternative Hallén formulation for case (c) with $k=0.005, k a=10^{-8} ;$ a thin wire case $(a \ll 1)$. Virtually identical behavior is seen for case $(\mathrm{b})$. The coefficients are divided into two sets: the even-index coefficients are clustered in the upper set, and the odd-index coefficients in the lower set. Note that the even set demonstrates extremely rapid convergence for $0 \leq n \leq 50$, followed by a regime of slower convergence. We speculate this must be the result of a small-weight singularity in the thin-wire solutions located near the origin on the imaginary axis (associated with a corresponding singularity the kernel itself has at $\left(z-z^{\prime}\right)=2 a i$ ), which results in a decrease of the convergence rates for $n>50$; see Table 5.3.

in Table 5.3. For this case, yet again a very thin wire problem, we used the alternative Hallén method described in section 4.3. We see from Table 5.3 that, for example, single precision accuracy results from this method for $N=40$; use of the regular Hallén and Pocklington methods of sections 4.1 and 4.2 requires much larger numbers of unknowns, of the order of $N=500$, to achieve comparable accuracies. Note that the condition number remains virtually constant throughout the table. A graphical solution $J$, consistent with our Figure 5.1(c), was given in [4] (case $a=1 e-6, \omega=0.01$ in that work). Errors of the order of $10^{-6}$ were reported in that reference from use of the hp-geometric Galerkin approach with 50 unknowns; Table 5.3 shows an accuracy of the order of $10^{-6}$ for $N=30$ unknowns. 
TABLE 5.4

Hallén and Pocklington solvers for case (d): $k=50, k a=1$. The current profile is shown in Figure 5.1(d). An N+1-term Chebyshev is used, and the algorithm is run with integration parameters $M_{1}$ and $M_{2}$ and splitting parameter $\Delta=5$. The quantity $C_{N}$ is the estimate of the reciprocal condition number provided by the linear algebra package LAPACK. The error oscillations are fully resolved: the maximum relative error $e_{\max }$ was obtained by evaluation of $J$ and $J_{\text {ref }}$ on a $2^{10}+1$-point Chebyshev grid; the reference solution $J_{\text {ref }}$ was obtained by means of the Hallén solver with $N=140, M_{1}=2^{12}$, and $M_{2}=2^{9}$. The evaluation of $J$ at the $2^{10}+1$-point Chebyshev grid required approximately $0.004 \mathrm{~s}$ of computational time in addition to the tabulated computing times. Note that in this case, in which the maximum value of the solution is of order $10^{-3}$, the solution errors are smaller than what might have been expected for the condition numbers reported in the table.

\begin{tabular}{ccccccccc}
\hline \multirow{2}{*}{$N$} & $M_{1}$ & $M_{2}$ & \multicolumn{3}{c}{ Hallén } & \multicolumn{3}{c}{ Pocklington } \\
\cline { 4 - 8 } & & & $e_{\max }$ & $T(\mathrm{~s})$ & $C_{N}$ & $e_{\max }$ & $T(\mathrm{~s})$ & $C_{N}$ \\
\hline 50 & $2^{6}$ & $2^{4}$ & $8.9 \cdot 10^{-2}$ & 0.04 & $8.8 \cdot 10^{-3}$ & $7.9 \cdot 10^{-1}$ & 0.05 & $9.7 \cdot 10^{-7}$ \\
60 & $2^{6}$ & $2^{5}$ & $3.4 \cdot 10^{-4}$ & 0.08 & $6.1 \cdot 10^{-3}$ & $3.3 \cdot 10^{-2}$ & 0.09 & $5.4 \cdot 10^{-7}$ \\
70 & $2^{7}$ & $2^{5}$ & $1.7 \cdot 10^{-7}$ & 0.15 & $4.8 \cdot 10^{-3}$ & $2.3 \cdot 10^{-5}$ & 0.16 & $3.7 \cdot 10^{-7}$ \\
80 & $2^{7}$ & $2^{5}$ & $4.9 \cdot 10^{-9}$ & 0.19 & $3.6 \cdot 10^{-3}$ & $2.9 \cdot 10^{-9}$ & 0.20 & $2.5 \cdot 10^{-7}$ \\
90 & $2^{7}$ & $2^{6}$ & $3.5 \cdot 10^{-10}$ & 0.31 & $2.8 \cdot 10^{-3}$ & $3.5 \cdot 10^{-10}$ & 0.32 & $1.8 \cdot 10^{-7}$ \\
100 & $2^{7}$ & $2^{6}$ & $9.5 \cdot 10^{-12}$ & 0.38 & $2.2 \cdot 10^{-3}$ & $9.5 \cdot 10^{-12}$ & 0.39 & $1.4 \cdot 10^{-7}$ \\
110 & $2^{7}$ & $2^{6}$ & $5.6 \cdot 10^{-12}$ & 0.45 & $1.8 \cdot 10^{-3}$ & $6.5 \cdot 10^{-12}$ & 0.46 & $1.0 \cdot 10^{-7}$ \\
120 & $2^{7}$ & $2^{6}$ & $3.9 \cdot 10^{-13}$ & 0.54 & $1.5 \cdot 10^{-3}$ & $4.5 \cdot 10^{-13}$ & 0.55 & $7.9 \cdot 10^{-8}$ \\
\hline
\end{tabular}

TABLE 5.5

Same as Table 5.4, with the following exceptions: $k=0.5, k a=10^{-2}$; the current profile is shown in Figure 5.1(e); the maximum value of the solution is of order 0.3 -the errors are consistent with the condition numbers reported, although in the Pocklington case they are smaller than what might have been expected in view of the tabulated condition numbers.

\begin{tabular}{ccccccccc}
\hline$N$ & $M_{1}$ & $M_{2}$ & \multicolumn{3}{c}{ Hallén } & \multicolumn{3}{c}{ Pocklington } \\
\cline { 4 - 8 } & & & $e_{\max }$ & $T(\mathrm{~s})$ & $C_{N}$ & $e_{\max }$ & $T(\mathrm{~s})$ & $C_{N}$ \\
\hline 10 & $2^{3}$ & $2^{2}$ & $3.6 \cdot 10^{-3}$ & 0.003 & $4.6 \cdot 10^{-2}$ & $3.6 \cdot 10^{-3}$ & 0.004 & $3.2 \cdot 10^{-4}$ \\
20 & $2^{3}$ & $2^{3}$ & $2.3 \cdot 10^{-4}$ & 0.005 & $1.2 \cdot 10^{-2}$ & $2.3 \cdot 10^{-4}$ & 0.006 & $3.8 \cdot 10^{-5}$ \\
30 & $2^{4}$ & $2^{4}$ & $2.3 \cdot 10^{-5}$ & 0.010 & $5.7 \cdot 10^{-3}$ & $2.3 \cdot 10^{-5}$ & 0.012 & $1.1 \cdot 10^{-5}$ \\
40 & $2^{5}$ & $2^{4}$ & $8.7 \cdot 10^{-7}$ & 0.020 & $5.1 \cdot 10^{-3}$ & $8.7 \cdot 10^{-7}$ & 0.022 & $4.8 \cdot 10^{-6}$ \\
50 & $2^{6}$ & $2^{4}$ & $1.9 \cdot 10^{-7}$ & 0.042 & $3.5 \cdot 10^{-3}$ & $1.9 \cdot 10^{-7}$ & 0.045 & $2.5 \cdot 10^{-6}$ \\
60 & $2^{6}$ & $2^{5}$ & $2.6 \cdot 10^{-8}$ & 0.076 & $2.4 \cdot 10^{-3}$ & $2.6 \cdot 10^{-8}$ & 0.079 & $1.5 \cdot 10^{-6}$ \\
70 & $2^{7}$ & $2^{5}$ & $7.2 \cdot 10^{-10}$ & 0.149 & $1.8 \cdot 10^{-3}$ & $7.2 \cdot 10^{-10}$ & 0.154 & $9.3 \cdot 10^{-7}$ \\
80 & $2^{7}$ & $2^{5}$ & $3.0 \cdot 10^{-10}$ & 0.191 & $1.4 \cdot 10^{-3}$ & $3.0 \cdot 10^{-10}$ & 0.197 & $6.3 \cdot 10^{-7}$ \\
90 & $2^{7}$ & $2^{5}$ & $3.4 \cdot 10^{-11}$ & 0.237 & $1.1 \cdot 10^{-3}$ & $3.4 \cdot 10^{-11}$ & 0.246 & $4.4 \cdot 10^{-7}$ \\
100 & $2^{7}$ & $2^{6}$ & $1.5 \cdot 10^{-12}$ & 0.379 & $8.8 \cdot 10^{-4}$ & $1.5 \cdot 10^{-12}$ & 0.389 & $3.2 \cdot 10^{-7}$ \\
\hline
\end{tabular}

Case (d) $k a=1$ and $k=50$. The current profile is shown in Figure 5.1(d), and numerical results for the regular Hallén and Pocklington methods of sections 4.1 and 4.2 are presented in Table 5.4. Both methods produce errors of the order of $10^{-7}$ for $N=70$, and yield double precision accuracy for $N=120$. The hp-geometric Galerkin approach of [4] (case $a=0.01, \omega=100$ in that work) yields results with an accuracy of $10^{-5}$ using a number of the order $N=350$ unknowns.

Case (e) $k a=10^{-2}$ and $k=0.5$. The current profile is shown in Figure 5.1(e), and numerical results for the regular Hallén and Pocklington methods of sections 4.1 and 4.2 are presented in Table 5.5. Both methods produce virtually identical results in this case, with rapid convergence in $N$ to nearly double precision accuracy. In particular, Table 5.5 shows results with errors of $10^{-5}$ and $10^{-6}$ for $N=30$ and 
TABLE 5.6

Same as Table 5.4, with the following exceptions: $k=0.005, k a=10^{-4}$; the current profile is shown in Figure 5.1(f); the reference solution $J_{\text {ref }}$ was obtained by means of the Pocklington solver with $N=140, M_{1}=2^{12}$, and $M_{2}=2^{9}$; the maximum value of the solution is of order 0.3 - the detailed discussion of conditioning and errors is given in the text.

\begin{tabular}{ccccccccc}
\hline \multirow{2}{*}{$N$} & $M_{1}$ & $M_{2}$ & \multicolumn{3}{c}{ Hallén } & \multicolumn{3}{c}{ Pocklington } \\
\cline { 3 - 8 } & & & $e_{\max }$ & $T(\mathrm{~s})$ & $C_{N}$ & $e_{\max }$ & $T(\mathrm{~s})$ & $C_{N}$ \\
\hline 10 & $2^{3}$ & $2^{2}$ & $3.6 \cdot 10^{-3}$ & 0.003 & $4.7 \cdot 10^{-2}$ & $3.6 \cdot 10^{-3}$ & 0.004 & $3.5 \cdot 10^{-4}$ \\
20 & $2^{3}$ & $2^{3}$ & $2.3 \cdot 10^{-4}$ & 0.005 & $1.2 \cdot 10^{-2}$ & $2.3 \cdot 10^{-4}$ & 0.006 & $4.3 \cdot 10^{-5}$ \\
30 & $2^{4}$ & $2^{4}$ & $2.3 \cdot 10^{-5}$ & 0.010 & $8.7 \cdot 10^{-3}$ & $2.3 \cdot 10^{-5}$ & 0.012 & $1.3 \cdot 10^{-5}$ \\
40 & $2^{5}$ & $2^{4}$ & $8.9 \cdot 10^{-7}$ & 0.020 & $5.1 \cdot 10^{-3}$ & $8.9 \cdot 10^{-7}$ & 0.022 & $5.4 \cdot 10^{-6}$ \\
50 & $2^{6}$ & $2^{4}$ & $1.9 \cdot 10^{-7}$ & 0.042 & $3.5 \cdot 10^{-3}$ & $1.9 \cdot 10^{-7}$ & 0.045 & $2.8 \cdot 10^{-6}$ \\
60 & $2^{6}$ & $2^{5}$ & $2.8 \cdot 10^{-8}$ & 0.076 & $2.5 \cdot 10^{-3}$ & $2.7 \cdot 10^{-8}$ & 0.079 & $1.6 \cdot 10^{-6}$ \\
70 & $2^{7}$ & $2^{5}$ & $8.5 \cdot 10^{-10}$ & 0.149 & $1.8 \cdot 10^{-3}$ & $7.2 \cdot 10^{-10}$ & 0.154 & $1.0 \cdot 10^{-6}$ \\
80 & $2^{7}$ & $2^{5}$ & $5.2 \cdot 10^{-10}$ & 0.191 & $1.4 \cdot 10^{-3}$ & $3.1 \cdot 10^{-10}$ & 0.197 & $6.9 \cdot 10^{-7}$ \\
90 & $2^{7}$ & $2^{5}$ & $1.5 \cdot 10^{-9}$ & 0.237 & $1.1 \cdot 10^{-3}$ & $4.8 \cdot 10^{-11}$ & 0.246 & $4.9 \cdot 10^{-7}$ \\
100 & $2^{7}$ & $2^{6}$ & $1.4 \cdot 10^{-9}$ & 0.379 & $8.9 \cdot 10^{-4}$ & $1.2 \cdot 10^{-11}$ & 0.389 & $3.6 \cdot 10^{-7}$ \\
\hline
\end{tabular}

$N=40$, respectively. The hp-geometric Galerkin approach of [4] (case $a=0.01$, $\omega=1$ in that work) yields results with an accuracy of $10^{-4}$ using a number of the order $N=40$ unknowns.

Case (f) $k a=10^{-4}$ and $k=0.005$. The current profile is shown in Figure 5.1(f), and numerical results for both methods are presented in Table 5.6. This is the only case in which we have found that the regular Pocklington method is clearly superior to the Hallén method. While both methods exhibit rapid convergence, the Hallén approach appears to break down beyond a relative error $\mathcal{O}\left(10^{-9}\right)$, while the relative error of the Pocklington method continues to decrease to $\mathcal{O}\left(10^{-11}\right)$. The reason for this is subtle: the current generated by the Hallén method relies on a superposition of solutions, shown in (4.4). We have noted that, under some circumstances, a subtractive cancellation occurs here that limits the accuracy obtainable using finite precision arithmetic. In view of these considerations, in this table, unlike all others, we use the results provided by the Pocklington method to produce the reference solution $J_{\text {ref }}$. A graphical solution $J$, consistent with our Figure 5.1(f), was given in [4] (case $a=0.01$, $\omega=0.01$ in that work), but errors were not reported in that reference for this case.

5.2. Conclusions. In contrast to published reports, we have found our Hallén and Pocklington approaches numerically equivalent in many situations. For very thin wires, the alternative method developed in section 4.3 (which gives rise to higher meshdensities at the end-points than those implicit in the Chebyshev method of sections 4.1 and 4.2) results in significant efficiency improvements. The numerical results we presented show that, in many cases, to achieve a given accuracy, the numbers $N$ of unknowns required by our codes are up to five times smaller than those required by the best solvers previously available. The short computing times demonstrated in section 5.1 arise not only from use of small numbers of unknowns but also from the small numbers $M_{1}$ and $M_{2}$ of integration points required for construction of the discretized operator. Unfortunately, timings have not been reported previously in the literature. In view of the superalgebraic convergence in the numbers $M_{1}$ and $M_{2}$ (see point (d) in the introduction) and the small numbers $N$ of unknowns required by the present solvers, we suggest that these methods should be significantly faster than others available previously. 
Appendix A. Asymptotic expansion of the kernel. We present the exact polynomials required to evaluate the asymptotic form of the thin-wire kernel given in (2.20) to order $M=10$.

$$
\begin{gathered}
\mathcal{A}_{0}(x)=1, \quad \mathcal{A}_{1}(x)=x, \quad \mathcal{A}_{2}(x)=x^{2}+48, \quad \mathcal{A}_{3}(x)=x\left(x^{2}+1620\right) \\
\mathcal{A}_{4}(x)=x^{4}+13440 x^{2}+241920, \quad \mathcal{A}_{5}(x)=x\left(x^{4}+63000 x^{2}+30240000\right) \\
\mathcal{A}_{6}(x)=x^{6}+213840 x^{4}+748440000 x^{2}+9580032000 \\
\mathcal{A}_{7}(x)=x\left(x^{6}+588588 x^{4}+8899450560 x^{2}+2669835168000\right) \\
\mathcal{A}_{8}(x)=x^{8}+1397760 x^{6}+67805337600 x^{4} \\
+136695560601600 x^{2}+1464595292160000 \\
\mathcal{A}_{9}(x)=x\left(x^{8}+2974320 x^{6}+381093672960 x^{4}\right. \\
\left.+3137163115806720 x^{2}+726029178229555200\right) \\
\mathcal{A}_{10}(x)=x^{10}+5814000 x^{8}+1714199760000 x^{6}+43444678717440000 x^{4} \\
+63863677714636800000 x^{2}+613091306060513280000
\end{gathered}
$$

$$
\mathcal{B}_{0}(x)=1, \quad \mathcal{B}_{1}(x)=x, \quad \mathcal{B}_{2}(x)=x^{2}+180, \quad \mathcal{B}_{3}(x)=x\left(x^{2}+2240\right),
$$$$
\mathcal{B}_{4}(x)=x^{4}+12600 x^{2}+1209600, \quad \mathcal{B}_{5}(x)=x\left(x^{4}+47520 x^{2}+49896000\right),
$$

$$
\begin{gathered}
\mathcal{B}_{6}(x)=x^{6}+140140 x^{4}+762810048 x^{2}+54486432000 \\
\mathcal{B}_{7}(x)=x\left(x^{6}+349440 x^{4}+6780533760 x^{2}+4881984307200\right), \\
\mathcal{B}_{8}(x)=x^{8}+771120 x^{6}+42343741440 x^{4} \\
+149388719800320 x^{2}+8963323188019200 \\
\mathcal{B}_{9}(x)=x\left(x^{8}+1550400 x^{6}+205703971200 x^{4}\right. \\
\left.+2482553069568000 x^{2}+1419192838103040000\right)
\end{gathered}
$$

Appendix B. Numerical evaluation of the $A_{n}$ and $B_{n}$ functions. In this section we discuss the efficient computation of the quantities $A_{n}$ and $B_{n}$ in (4.2) and (4.14), respectively. The numerical evaluation of these functions is complicated not only by the logarithmic singularity of the kernel at $z=t$ (resp., $\rho=0$ ) but also by an interesting near-singular behavior of the kernel functions $F_{1}$ and $F_{2}$ in a neighborhood of the singularity. As the wire radius $a$ decreases, the real part of each of the kernel functions $F_{1}$ and $F_{2}$ on the domain $[-1 / a, 1 / a]$ contains increasingly rapid variations, developing a sharp peak in a neighborhood of $z-t=\mathcal{O}(a)$. This behavior, which is closely related to the complex singularities mentioned in section 4.3 , is depicted in Figure B.1 for the function $g_{r 1}(\rho)$-which is related to the dimensional kernel function $F_{1}(z-t)$ by (2.19). Qualitatively similar behavior is exhibited by the function $F_{2}$. While these functions remain analytic, their resolution with an evenly spaced FFT may require prohibitively large numbers of sampling points. In what follows we outline our procedure for simultaneously treating the unusual combination in the kernel of a logarithmic singularity and the sharp peaks discussed above. 


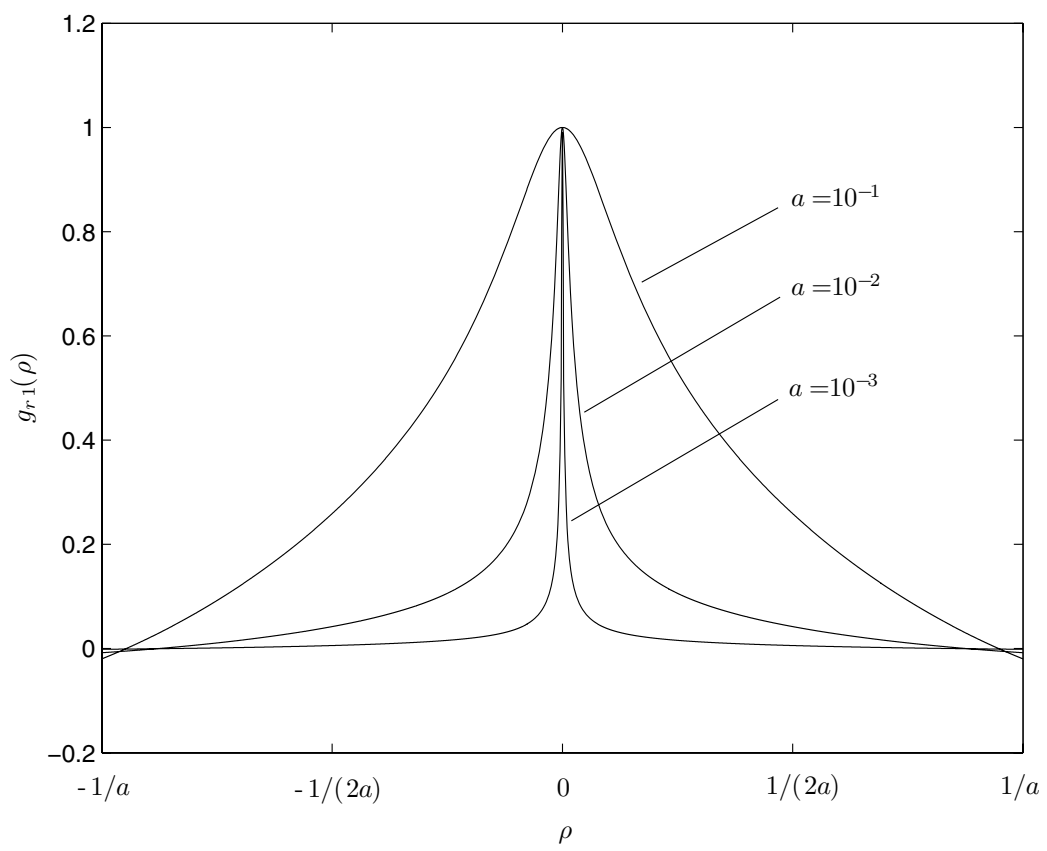

FIG. B.1. The nondimensional kernel function $g_{r 1}(\rho)$ for $k=1$ and $a=10^{-1}, a=10^{-2}$ and $a=10^{-3}$. Both kernel functions $g_{r 1}(\rho)$ and $g_{r 2}(\rho)$ exhibit a sharp peak in the neighborhood $\rho=\mathcal{O}(1)$.

B.1. Evaluation of the functions $\boldsymbol{A}_{\boldsymbol{n}}$. In what follows we are concerned with the evaluation of the integrals

$$
A_{n}(z)=\mathcal{H}\left\{T_{n}\right\}(z)=\int_{-1}^{1}\left[F_{1}(z-t) \ln |z-t|+F_{2}(z-t)\right] \frac{T_{n}(t)}{\sqrt{1-t^{2}}} d t .
$$

Since $G(z)$ is an even function of $z$, it easy to verify that the functions $A_{n}$ satisfy

$$
A_{n}(-z)=(-1)^{n} A_{n}(z)
$$

so that, although the values of the $A_{n}$ 's in the full interval $z \in[-1,1]$ are required by our method, actual integrations to produce these functions numerically need only be performed for $z \in[0,1]$.

To evaluate the functions $A_{n}$ on a Chebyshev grid (see Remark 4.1) and to eliminate the end-point singularities in (B.1) we introduce the substitutions $z=\cos \alpha$ and $t=\cos \theta$, which reduce the corresponding integrals to

$$
A_{n}(\cos \alpha)=\int_{0}^{\pi} G(\cos \alpha-\cos \theta) \cos n \theta d \theta
$$

The irregular features in the integrand of (B.3), which arise from corresponding features of $F_{1}$ and $F_{2}$, can be resolved with a reasonable expense by means of a local integration scheme using a fine grid on the domain $|z-t| \leq 2 a \Delta$, where $\Delta$ is the splitting parameter defined in section 2.3. Outside this domain, integration on a much coarser grid is sufficient to achieve the desired accuracy. 
In detail, we partition the integration domain $[0, \pi]$ into two regions, the fine- and coarse-discretization domains, that correspond to regions of fast and slow variations of the integrand in (B.1) and (B.3). To define the fine-discretization region, in most cases it is sufficient to restrict the angular coordinate in (B.3) to the domain $\theta \in\left[\theta_{1}, \theta_{2}\right]$, with $\theta_{1}=\cos ^{-1}(z+2 a \Delta)$ and $\theta_{2}=\cos ^{-1}(z-2 a \Delta)$. One notable exception occurs when $z+2 a \Delta>1$; this special case is treated by setting $\theta_{1}=0$. The analogous case, when $z-2 a \Delta<1$, does not occur in our method, since values of (B.3) for $z \in[-1,0$ ) are obtained by the symmetry relation (B.2). Setting $\varepsilon_{1}=\alpha-\theta_{1}$ and $\varepsilon_{2}=\theta_{2}-\alpha$, the domain of integration is thus partitioned as

$$
[0, \pi] \equiv\left[0, \alpha-\varepsilon_{1}\right] \cup\left[\alpha-\varepsilon_{1}, \alpha\right] \cup\left[\alpha, \alpha+\varepsilon_{2}\right] \cup\left[\alpha+\varepsilon_{2}, \pi\right] .
$$

Owing to (B.2), we have the restriction $\alpha \in[0, \pi / 2]$. In the fine-discretization region $\theta \in\left[\alpha-\varepsilon_{1}, \alpha+\varepsilon_{2}\right]$ we develop a special integration method which treats the logarithmic singularity of $G$. Outside of this region, different methods are applied on a coarse grid.

Integration in the coarse-discretization region $\theta \in\left[0, \alpha-\varepsilon_{1}\right] \cup\left[\alpha+\varepsilon_{2}, \pi\right]$. In this region, which lies away from the $\theta=\alpha$ singularity, the kernel $G$ is smooth and contributions to the $A_{n}$ functions are computed by means of an FFT-based $M_{1}+1$ point Clenshaw-Curtis integration rule on each of the two relevant subintervals.

Integration in the fine-discretization region $\theta \in\left[\alpha-\varepsilon_{1}, \alpha+\varepsilon_{2}\right]$. We illustrate the method of computation of contributions to $A_{n}(\cos \alpha)$ for the left-hand interval $\theta \in\left[\alpha-\varepsilon_{1}, \alpha\right]$; results for the right-hand interval $\theta \in\left[\alpha, \alpha+\varepsilon_{2}\right]$ are obtained in an analogous fashion. We map $x \in[-1,1]$ to $\theta \in\left[\alpha-\varepsilon_{1}, \alpha\right]$ with the change of variables $\theta=\varepsilon_{1}(x-1) / 2+\alpha$, and for each $n$, we introduce the approximation

$$
F_{1}(\cos \alpha-\cos \theta) \cos n \theta \approx \frac{1}{2} e_{0}^{(n)}(\alpha)+\sum_{m=1}^{M_{2}} e_{m}^{(n)}(\alpha) T_{m}(x) ; \quad \theta=\theta(x) .
$$

A similar expansion of length $M_{2}+1$ for $F_{2}(\cos \alpha-\cos \theta) \cos n \theta$ is introduced. The quantity $M_{2}+1$, which is independent of $M_{1}$, is the length of the fast cosine transform used to compute the series coefficients in this region. The integrals involving $F_{2}$ are computed using an FFT-based $M_{2}+1$-point Clenshaw-Curtis integration rule. In view of (1.5), the integral involving $F_{1}$ possesses a logarithmic singularity at $x=1$ which, for high-order integration, requires special treatment. The method we use to resolve this singularity integrates the logarithmic terms exactly: For $\alpha \neq 0$ and $\alpha=0$ we use the identities

$$
\ln |\cos \alpha-\cos \theta|=\ln \left|\frac{\cos \alpha-\cos \theta}{x-1}\right|+\ln |x-1|
$$

and

$$
\ln |1-\cos \theta|=\ln \left|\frac{1-\cos \theta}{(x-1)^{2}}\right|+2 \ln |x-1|,
$$

respectively. The Taylor expansion

$$
\cos \alpha-\cos \theta=\frac{\varepsilon_{1}}{2}(x-1) \sin \alpha+\frac{\varepsilon_{1}^{2}}{8}(x-1)^{2} \cos \alpha+\mathcal{O}\left(\varepsilon_{1}^{3}(x-1)^{3}\right)
$$

then shows that the first logarithmic term on the right-hand side of each of (B.6) and (B.7) is regular, so that the integrals involving those terms may be computed with 
high-order accuracy by means of the $M_{2}+1$-point Clenshaw-Curtis integration rule. The singular terms are integrated using the rule

$$
\int_{-1}^{1} F_{1}(\cos \alpha-\cos \theta) \cos n \theta \ln |x-1| d x \approx \frac{1}{2} w_{0} e_{0}^{(n)}(\alpha)+\sum_{m=1}^{M_{2}} w_{m} e_{m}^{(n)}(\alpha),
$$

where $w_{m}$ are the integration weights

$$
w_{m}=\int_{-1}^{1} T_{m}(t) \ln |1-t| d t .
$$

A closed-form expression for the weights $w_{m}$ may be derived by making the substitution $t=\cos 2 \psi$ in (B.10), expanding the terms $\cos m \psi$ in powers of $\cos \psi$, and applying the identity $[6,4.387]$ on a term-by-term basis. This leads to the results $w_{0}=2 \ln 2-2, w_{1}=-1$, and, for $m>1$,

$$
\begin{aligned}
w_{m}= & \frac{\left[1+(-1)^{m}\right] \ln 2}{1-m^{2}} \\
& -2 m \sum_{n=0}^{m} \frac{(-1)^{n} 2^{2 m-2 n}}{(m-n+1)(2 m-n)}\left(\begin{array}{c}
2 m-n \\
n
\end{array}\right)[\gamma+\Psi(m-n+2)],
\end{aligned}
$$

where $\gamma=0.57721566 \ldots$ is Euler's constant. In our implementation, the weights (B.11) have been precomputed to machine precision for all values of $m$ up to $m=2^{9}$ and saved in a data file which is read into memory every time the code is executed. The logarithmic integrals over the subinterval $\theta \in\left[\alpha, \alpha+\varepsilon_{2}\right]$ are computed in a similar manner, with integration weights that can be obtained from the data set above, namely,

$$
\int_{-1}^{1} T_{m}(t) \ln |1+t| d t=(-1)^{m} w_{m}
$$

B.2. Integration for the alternative Hallén method. We now consider the efficient evaluation of the $B_{n}$ functions defined in (4.14); these must obey the symmetry relations

$$
B_{n}(w+\pi / 2)=(-1)^{n} B_{n}(w), \quad w \in[0, \pi / 2] ;
$$

compare with (B.2). Thus, numerical integration of the $B_{n}$ functions need only be performed for uniformly spaced values of $w \in[0, \pi / 2]$. The integration of the $B_{n}$ functions follows in close analogy to the treatment of the $A_{n}$ functions, outlined in Appendix B.1.

Appendix C. Computation of the total current. As mentioned in section 5, the accurate evaluation of the total current $J(z)=I(z) / \sqrt{1-z^{2}}$ from the reduced current $I(z)$ requires special consideration: clearly the error in the current obtained by straightforward division tends to infinity as the evaluation points tend toward the end-points. To resolve this difficulty it is convenient to rewrite $J(z)$ in terms of a trigonometric series, and avoid the explicit division by the quantity $\sqrt{1-z^{2}}$. In what follows, we derive a representation for the total current which is used to evaluate currents arising from all three methods of solution presented in this paper. 
With the substitution $z=\cos \theta$ in (3.1) and (4.1), an approximation for the total current is readily derived by exploiting the orthogonality of the trigonometric functions:

$$
J(\cos \theta) \approx \sum_{n=1}^{N+1} c_{n} \sin (n \theta) .
$$

The coefficients in (C.1) are given in terms of the $b_{n}$ coefficients in (4.1) by $c_{1}=b_{0}$, $c_{2}=2 b_{1}$, and

$$
c_{n+1}=2 b_{n}+c_{n-1}, \quad 2 \leq n \leq N .
$$

The coefficients $c_{n}$ for $n \geq 2$ are easily found by forward recursion using (C.2). Accurate values of the total current on a fine Chebyshev grid (which is necessary to obtain maximum relative errors owing to fast error oscillations) are obtained by means of a zero-padded fast-sine-evaluation of the series (C.1).

In the context of the very thin wire algorithm, in turn, it is possible to derive a formula analogous to (C.1) for the current $J$ in the $w$ variables to produce this quantity everywhere in the wire. However, the current $J$ in the $w$ variables vanishes quadratically at the end-points, while it only vanishes linearly at those points in the $\theta$ variables. The vanishing order at the end-points is related to ill-conditioning in evaluation of the $J$ function: evaluation of quadratically vanishing quantities on the basis of cosine coefficients of order 1 is clearly more ill-conditioned than evaluation of corresponding quantities that only vanish linearly at the end-points. Thus, higher accuracy can be extracted for $J$ if the reduced current function $I$ in $w$-space is first transformed to $\theta$-space and then a sine series of $J$ in $\theta$ is used as earlier in this section. In detail, given the $N$-term cosine series for the reduced current in (4.12), values of $I$ are computed at the set of $N_{2}$ Chebyshev points (see (4.3)) for $N_{2} \gg N-$ which can be accomplished by an appropriately zero-padded FFT, together with loworder polynomial interpolation. For the results presented in this work, we have used $N_{2}=2^{10}+1$. Using this data the $b_{n}$ coefficients of the Chebyshev series (4.1) can be obtained, and can then be used to compute the $c_{n}$ coefficients of the sineseries representation for the total current in (C.1). This method produces uniformly accurate solutions throughout the wire for the very thin wire problem-including around the wire end-points. Note that the supersampling procedure described here is computationally inexpensive (as supported by data in the relevant table captions), since it can be accomplished by means of an FFT.

\section{REFERENCES}

[1] M. Abramowitz and I. A. Stegun, eds., Handbook of Mathematical Functions with Formulas, Graphs, and Mathematical Tables, Dover, New York, 1992. Reprint of the 1972 edition.

[2] R. A. Adams, Sobolev Spaces, Pure Appl. Math. 65, Academic Press, New York, 1975.

[3] P. F. Byrd and M. D. Friedman, Handbook of Elliptic Integrals for Engineers and Scientists, 2nd ed., revised, Grundlehren Math. Wiss. 67, Springer-Verlag, New York, 1971.

[4] P. J. Davies, D. B. Duncan, and S. A. Funken, Accurate and efficient algorithms for frequency domain scattering from a thin wire, J. Comput. Phys., 168 (2001), pp. 155-183.

[5] G. FIKIORIS, The approximate integral equation for a cylindrical scatterer has no solution, J. Electromagn. Waves Appl., 15 (2001), pp. 1153-1159.

[6] I. S. Gradshteyn and I. M. Ryzhik, Table of Integrals, Series, and Products, 6th ed., Academic Press, San Diego, CA, 2000. Translated from the Russian; translation edited and with a preface by A. Jeffrey and D. Zwillinger. 
[7] D. S. Jones, Note on the integral equation for a straight wire antenna, Proc. IEE-H, 128 (1981), pp. 114-116.

[8] J. C. Mason And D. C. Handscomb, Chebyshev Polynomials, Chapman \& Hall/CRC, Boca Raton, FL, 2003.

[9] L. W. Pearson, A separation of the logarithmic singularity in the exact kernel of the cylindrical antenna integral equation, IEEE Trans. Antennas Propagat., AP-23 (1975), pp. 256-258.

[10] H. C. Pocklington, Electrical oscillations in wires, Proc. Cambridge Phil. Soc., 9 (1897), pp. $324-332$.

[11] J. B. READE, Asymptotic behaviour of eigenvalues of certain integral equations, Proc. Edinburgh Math. Soc. (2), 22 (1979), pp. 137-144.

[12] T. J. Rivlin, Chebyshev Polynomials: From Approximation Theory to Algebra and Number Theory, 2nd ed., Pure Appl. Math., John Wiley \& Sons, New York, 1990.

[13] B. P. RYnne, The well-posedness of the integral equations for thin wire antennas, IMA J. Appl. Math., 49 (1992), pp. 35-44.

[14] C. SNow, Hypergeometric and Legendre Functions with Applications to Integral Equations of Potential Theory, National Bureau of Standards Appl. Math. Ser. 19, U.S. Government Printing Office, Washington, D.C., 1952.

[15] G. T. Symm, An integral equation method in conformal mapping, Numer. Math., 9 (1966), pp. $250-258$.

[16] W.-X. WANG, The exact kernel for cylindrical antenna, IEEE Trans. Antennas Propagat., AP-39 (1991), pp. 434-435.

[17] Y. Yan And I. H. SlOAN, On integral equations of the first kind with logarithmic kernels, J. Integral Equations Appl., 1 (1988), pp. 549-579. 